\title{
Symmetry-protected topological phases in spinful bosons with a flat band
}

\author{
Hong Yang $\odot,{ }^{1, *}$ Hayate Nakano $\odot,{ }^{1}$ and Hosho Katsura $\oplus^{1,2,3}$ \\ ${ }^{1}$ Department of Physics, Graduate School of Science, The University of Tokyo, 7-3-1 Hongo, Bunkyo-ku, Tokyo 113-0033, Japan \\ ${ }^{2}$ Institute for Physics of Intelligence, The University of Tokyo, 7-3-1 Hongo, Bunkyo-ku, Tokyo 113-0033, Japan \\ ${ }^{3}$ Trans-scale Quantum Science Institute, The University of Tokyo, 7-3-1 Hongo, Bunkyo-ku, Tokyo 113-0033, Japan
}

(Received 25 January 2021; accepted 18 May 2021; published 14 June 2021)

\begin{abstract}
We theoretically demonstrate that interacting symmetry-protected topological (SPT) phases can be realized with ultracold spinful bosonic atoms loaded on the lattices which have a flat band at the bottom of the band structure. Ground states of such systems are not conventional Mott insulators in the sense that the ground states possess not only spin fluctuations but also non-negligible charge fluctuations. The SPT phases in such systems are determined by both spin and charge fluctuations at zero temperature. We find that the many-body ground states of such systems can be exactly obtained in some special cases, and these exact ground states turn out to serve as representative states of the SPT phases. As a concrete example, we demonstrate that spin- 1 bosons on a sawtooth chain can be in an SPT phase protected by $\mathbb{Z}_{2} \times \mathbb{Z}_{2}$ spin rotation symmetry or time-reversal symmetry, and this SPT phase is a result of spin fluctuations. We also show that spin-3 bosons on a kagome lattice can be in an SPT phase protected by $D_{2}$ point group symmetry, but this SPT phase is, however, a result of charge fluctuations.
\end{abstract}

DOI: 10.1103/PhysRevResearch.3.023210

\section{INTRODUCTION}

Symmetry-protected topological (SPT) phases refer to the quantum phases of those short-range entangled ground states that can never be smoothly deformed into product states while preserving certain symmetry. On the other hand, a ground state is classified into a trivial phase if it can be smoothly deformed into a product state even when certain symmetry is imposed [1]. A product state stands for a tensor product of microscopic states and possesses no quantum entanglement. In contrast, entanglement in the SPT phases cannot be smoothly eliminated when preserving the symmetry. The Affleck-Kennedy-Lieb-Tasaki (AKLT) models provide great insight into the SPT phases of interacting bosonic systems. The AKLT models are a class of quantum spin models that can be defined on arbitrary lattices [2,3]. The models have exact and unique ground states, known as the valance-bondsolid (VBS) states. In a simple one-dimensional (1D) chain, the spin-1 VBS state (i.e., the ground state of the spin-1 AKLT model) represents an SPT phase protected by any of the following symmetries [4-7]: (1) $\mathbb{Z}_{2} \times \mathbb{Z}_{2}$ spin rotation symmetry, (2) time-reversal symmetry, and (3) inversion symmetry. This SPT phase is often called the Haldane phase. This Haldane phase is also characterized by a nonlocal order parameter-the spin string order parameter, which quantifies the hidden antiferromagnetic order in the 1D spin-1 VBS

\footnotetext{
*yanghongphy@gmail.com

Published by the American Physical Society under the terms of the Creative Commons Attribution 4.0 International license. Further distribution of this work must maintain attribution to the author(s) and the published article's title, journal citation, and DOI.
}

state [5,7-9]. In certain two and higher dimensional lattices, integer-spin VBS states can be in SPT phases if either translation symmetry or crystalline symmetry is involved, as we will discuss later.

Ultracold atoms/molecules in optical lattices serve as an ideal platform for realizing topological quantum phases due to the high tunability of interactions, the viability of building various lattice structures, and the feasibility of directly measuring nonlocal order parameters [10,11]. Motivated by recent experimental progress, many theoretical predictions about the existence of the Haldane phase in lattice systems of bosons [12-23] and fermions [24-32] have been made.

Alkali-metal atoms carry integer spins and are thus often treated as spinful bosons in experiments [33]. Spinful bosons in optical lattices typically have both spin and charge degrees of freedom (DOF). Free from the Pauli exclusion principle, one major difficulty of theoretically studying the systems of many-body spinful bosons lies in their immense Hilbert spaces (i.e., a huge number of DOF). Therefore, except for very few rigorous results [34,35], various approximations or constraints have been employed to simplify the problem (i.e., to reduce the Hilbert space dimension by freezing some DOF). In particular, to theoretically investigate the Haldane phase of bosonic atoms in one dimension, there have been two main approaches. One is to study the effective spin Hamiltonians by focusing on the conventional Mott insulating limit where the charge DOF are frozen [23]. For example, the system of Mott insulating spin-1 bosons is effectively described by the bilinear-biquadratic (BLBQ) model, whose ground state in one dimension has been known to exhibit the Haldane phase in a wide parameter region [36]. The other approach is to study models that describe itinerant but spinless bosons. (A system is said to be itinerant if it has charge DOF.) In the itinerant case, it is 
generally believed that a sufficiently strong long-range (repulsive) interaction is indispensable for triggering the SPT phase [12-20,22]. The mechanism is as follows. At the filling of one spinless boson per site on average, if we truncate the particle number on each site to $n=0,1,2$, one can define pseudospin as $\mathcal{S}^{z}:=n-1$, thus resulting in an effective spin1 model, where the long-range repulsion acts as an anisotropic spin exchange interaction $[4,13]$. However, among bosonic alkali-metal atoms, although a relatively strong dipole-dipole interaction plays the role of long-range interaction in certain situations [33], the dipole-dipole interaction is usually much weaker than the short-range $s$-wave collision, and thus the long-range interaction is typically negligible in many experiments [33].

In short, despite the fact that itinerant, spinful, and short-range interacting bosonic atoms are very common in experiments, due to the difficulty of theoretically dealing with the huge amount of DOF, it remains an open question whether the SPT phases can be realized in such systems. Moreover, if the answer is yes, what kinds of SPT phases can we get? We address these issues and argue that, when there is a flat band at the bottom of the band structure (which we dub a bottom flat band), SPT phases can be realized with short-range interacting bosons that possess both unfrozen spin and charge DOF. As a result, the SPT phases in such systems are characterized by nontrivial spin or charge entanglement.

A flat band refers to an energy band that is independent of the quasimomentum. Usually, a flat band in an optical lattice is the highest band. However, by shaking the optical lattices, one can invert the sign of hopping [37-42], and the flat band thus becomes the lowest band. Such lattice shaking techniques have been realized experimentally [43-47].

Single-body eigenstates of a flat band can usually be chosen to be strictly localized on a finite number of lattice sites. Such eigenstates are termed as compact localized states (CLSs) [48,49]. Different CLSs reside in different patches (regions) of the lattice. Short-range interaction ( $s$-wave collision) between two bosons can happen only when their wave functions have a finite overlap. (This is natural, because the short-range interaction does not occur unless two particles are very close to each other.) At low temperatures, boson wave functions tend to avoid overlapping each other in order to lower the system's energy. Let $X$ be a $d$-dimensional lattice with a bottom flat band and $N$ unit cells. When $N$ spin- $f$ bosons are loaded on $X$, the wave function overlaps can be minimized if each of the $N$ CLSs hosts a boson. In other words, $N$ bosons are distributed into $N$ different patches. A boson is free to move around within a patch, which gives rise to charge fluctuations in the ground state. On the other hand, since all the patches (CLSs) are occupied by bosons (i.e., the whole lattice is fully "packed" with bosons), partial overlaps between neighboring wave functions are inevitable. We notice an analogy between the Hamiltonian that describes the short-range $s$-wave collision among spin- $f$ bosons and the spin- $f$ AKLT Hamiltonian. This analogy implies that the wave function overlaps will not cost energy, if the spins of bosons entangle in a clever way similar to a spin- $f$ VBS state. (Intuitively, since the $s$-wave collision is spin-dependent by its nature, when the bosons are in a certain spin state, the collision between them can be avoided even if the bosons are very close to each other.) When certain parameters in the Hamiltonian are fine-tuned, the above configuration (lattice fully packed with CLSs) becomes the exact and unique ground state, and the state turns out to serve as a representative state of the symmetry-protected phases of the system. (In this paper, the term "symmetry-protected phase" refers to either SPT or trivial phase.) We find that the phases are determined by the spin or charge fluctuations in the ground state. In this paper, we find a large class of models whose ground states can be exactly written down when certain parameters are properly chosen. Each model has several on-site and crystalline symmetries. Depending on the symmetry, these exact ground states can be in either SPT or trivial phases. In particular, in terms of crystalline symmetries, charge fluctuations can play a nontrivial role.

This paper will gradually build up a general framework on the SPT phases of spinful bosons with a flat band, starting from a simple 1D spin-1 model before progressing towards general dimensions and general spins. The remainder of this paper is divided into two parts: Sec. II and Sec. III. In Sec. II, we use spin-1 bosons on the 1D sawtooth chain as a concrete example to demonstrate our argument. The sawtooth chain has two energy bands, and the bottom one is flat. We prove that when the interaction between spin-1 bosons is fine-tuned, the ground state is unique and can be exactly obtained. The proof is based on the fact that the ground state can be exactly mapped to the 1D spin-1 VBS state. This exact ground state turns out to be in a Haldane phase. Beyond the fine-tuned case, based on perturbation theory and numerical calculations, we confirmed that the Haldane phase exists in a rather broad parameter region. In Sec. III, we discuss the SPT phases with a general setup: short-range interacting spin- $f$ bosons on a bottom-flat-band lattice $X$ in $d$ dimension. Let $\left|\mathrm{GS}_{f, X}\right\rangle$ be the many-body ground state. Let $\left|\mathrm{VBS}_{f, X^{\prime}}\right\rangle$ be the spin- $f$ VBS state defined on a lattice $X^{\prime}$ (i.e., the ground state of the spin- $f$ AKLT model on $X^{\prime}$ ). With fine-tuned interactions, $\left|\mathrm{GS}_{f, X}\right\rangle$ can be exactly mapped to $\left|\mathrm{VBS}_{f, X^{\prime}}\right\rangle$, provided that the lattice structures of $X$ and $X^{\prime}$ satisfy a certain relation. This proves that $\left|G S_{f, X}\right\rangle$ is the exact and unique ground state of the itinerant spin- $f$ model. The spin fluctuations of $\left|\mathrm{GS}_{f, X}\right\rangle$ are inherited from $\left|\mathrm{VBS}_{f, X^{\prime}}\right\rangle$. Therefore, with respect to the spin rotation symmetry or the combination of spin rotation and translation symmetry, the $d$-dimensional symmetry-protected phase of $\left|\mathrm{GS}_{f, X}\right\rangle$ is identical to that of $\left|\mathrm{VBS}_{f, X^{\prime}}\right\rangle$. Spins in $\left|\mathrm{VBS}_{f, X^{\prime}}\right\rangle$ are pinned to the lattice sites and cannot move. However, $\left|G_{f, X}\right\rangle$ is not a conventional Mott-insulating state (where a fixed number of bosons stay rigidly on each site), i.e., spin- $f$ bosons in $\left|\mathrm{GS}_{f, X}\right\rangle$ have nonvanishing charge fluctuations. It turns out that in terms of crystalline symmetries (i.e., point group or space group symmetries), both spin and charge fluctuations in $\left|\mathrm{GS}_{f, X}\right\rangle$ together determine its symmetry-protected phase. Hence, the crystalline-symmetry-protected phases of $\left|\mathrm{GS}_{f, X}\right\rangle$ and $\left|\mathrm{VBS}_{f, X^{\prime}}\right\rangle$ may not be identical, because the charge fluctuations may play a nontrivial role in the former state. For example, as we will show later, interacting spin-3 bosons in the kagome lattice can be in an SPT phase protected by the point group $D_{2}$ or $D_{3}$, and this SPT phase is purely a consequence of charge fluctuations at zero temperature. 


\section{SPIN-1 BOSONS ON A SAWTOOTH CHAIN: AN EXAMPLE}

Let us start from a simple but nontrivial model: the spin-1 Bose-Hubbard model on the sawtooth chain (BHMSC). In Sec. II A, we introduce the spin-1 BHMSC and the 1D spin1 bilinear-biquadratic (BLBQ) model. The ground state of the BLBQ model is exactly solvable at the AKLT point. In Sec. II B, we prove that in a special case where the interaction between spin-1 bosons is fine-tuned, the ground state sectors of the spin-1 BHMSC and the AKLT model can be exactly mapped to each other, which enables us to obtain an exact and unique ground state of the spin-1 BHMSC. This ground state, as we will show in Sec. II C, turns out to serve as a representative state of the Haldane phase. We find that the Haldane phase in this itinerant spin-1 boson system, characterized by both nonvanishing spin and charge string order parameters, is protected by (1) $\mathbb{Z}_{2} \times \mathbb{Z}_{2}$ symmetry or (2) time-reversal symmetry, but not (3) inversion symmetry. In Sec. II D, perturbation theory builds another bridge between the spin-1 BHMSC and the BLBQ model. In Sec. IIE, the phase diagram of the spin-1 BHMSC is investigated with numerical calculations based on the variational uniform matrix product state (VUMPS) algorithm [50,51], which suggests that the system can be in either a gapped Haldane or gapless critical phase.

\section{A. Hamiltonian}

For spin-1 bosons (such as ${ }^{7} \mathrm{Li},{ }^{23} \mathrm{Na},{ }^{41} \mathrm{~K}$, etc.) in a lattice system, let $\hat{a}_{r, \alpha}^{\dagger}\left(\hat{a}_{r, \alpha}\right)$ be the operator that creates (annihilates) a boson at lattice site $r$ with magnetic sublevel $\alpha=-1,0,1$. The on-site spin operator $\hat{\boldsymbol{S}}_{r}=\left(\hat{S}_{r}^{x}, \hat{S}_{r}^{y}, \hat{S}_{r}^{z}\right)$ is defined as $\hat{S}_{r}^{z}:=$ $\sum_{\alpha, \beta} \hat{a}_{r, \alpha}^{\dagger} S_{\alpha, \beta}^{z} \hat{a}_{r, \beta}$ with $S_{\alpha, \beta}^{z}=\alpha \delta_{\alpha, \beta}$ being the $z$-component of the spin matrix for spin-1 (and similar definitions for $\hat{S}_{r}^{x}$ and $\hat{S}_{r}^{y}$ ). We also define $\hat{n}_{r}:=\sum_{\alpha} \hat{a}_{r, \alpha}^{\dagger} \hat{a}_{r, \alpha}$, which counts the particle number on site $r$. Spin-1 atoms in optical lattices are effectively described by the spin-1 Bose-Hubbard model $[52,53]$ :

$$
\begin{aligned}
\hat{H} & =\hat{H}_{\text {hop }}+\hat{H}_{\text {int }}, \\
\hat{H}_{\text {hop }} & =-\sum_{\left\langle r, r^{\prime}\right\rangle} \sum_{\alpha=-1}^{1} t_{r, r^{\prime}} \hat{a}_{r, \alpha}^{\dagger} \hat{a}_{r^{\prime}, \alpha}+\sum_{r} V_{r} \hat{n}_{r}, \\
\hat{H}_{\text {int }} & =\sum_{r}\left(g_{0, r} \hat{P}_{r}^{(0)}+g_{2, r} \hat{P}_{r}^{(2)}\right),
\end{aligned}
$$

where $\hat{H}_{\text {hop }}$ is the single-body Hamiltonian which contains both hopping and on-site potential terms, and $\hat{H}_{\text {int }}$ describes the interactions ( $s$-wave collisions) between spin-1 bosonic atoms $[33,54]$. There are two kinds of interactions: $\hat{P}_{r}^{(S)}$ stands for the projection operator onto the state with total spin $S=0,2$ for a pair of spin-1 bosons at site $r$. For example, $\hat{P}_{r}^{(0)}=\hat{b}_{r}^{\dagger} \hat{b}_{r}$, where

$$
\hat{b}_{r}^{\dagger}:=\frac{1}{\sqrt{6}}\left(\hat{a}_{r, 0}^{\dagger} \hat{a}_{r, 0}^{\dagger}-2 \hat{a}_{r, 1}^{\dagger} \hat{a}_{r,-1}^{\dagger}\right)
$$

creates a spin singlet. $S=1$ is forbidden because two spin-1 bosons on the same site never form a total spin $S=1$ statesuch a spin state is antisymmetric. The projection operators
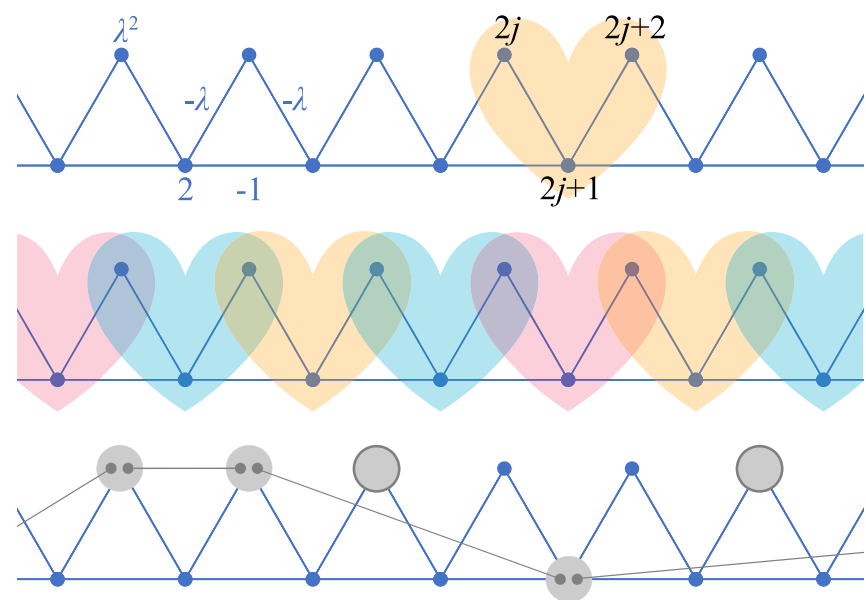

spin-1 boson

2-body spin singlet

FIG. 1. (Upper panel) The sawtooth lattice and one of its zeroenergy state. The state is localized on three consecutive sites covered by the heart shape. The blue characters indicate the values of $t_{r, r^{\prime}}$ and $V_{r}$. (Middle panel) The state $|\boldsymbol{\beta}\rangle$ in Eq. (12) with a typical choice of $\boldsymbol{\beta}$. Three different colors denote three different magnetic sublevels. Linearly independent CLSs cover the whole lattice, and two neighboring CLSs overlap on a top site. (Lower panel) The "hidden VBS order" illustrated by a typical component of $|\mathrm{GS}\rangle$ in Eq. (19).

can be explicitly expressed as $\hat{P}_{r}^{(0)}=\left[-\left(\hat{\boldsymbol{S}}_{r}\right)^{2}+\left(\hat{n}_{r}\right)^{2}+\hat{n}_{r}\right] / 6$ and $\hat{P}_{r}^{(2)}=\left[\left(\hat{\boldsymbol{S}}_{r}\right)^{2}+2\left(\hat{n}_{r}\right)^{2}-4 \hat{n}_{r}\right] / 6[33,54]$. The sum of them yields the "completeness relation":

$$
\hat{P}_{r}^{(0)}+\hat{P}_{r}^{(2)}=\frac{1}{2} \hat{n}_{r}\left(\hat{n}_{r}-1\right) .
$$

We assume the interaction strength $g_{S, r} \geqslant 0$ as is the case of long-lived alkali-metal spin-1 condensates [33]; $\hat{H}_{\text {int }}$ is thus positive semidefinite.

On a sawtooth chain (see Fig. 1) with $N$ unit cells $(2 N$ sites), the single-body Hamiltonian can be written in a compact form as [55-57]

$$
\hat{H}_{\text {hop }}=\hat{H}_{\text {saw }}=\sum_{i=1}^{N} \sum_{\alpha=-1}^{1} \hat{A}_{i, \alpha}^{\dagger} \hat{A}_{i, \alpha},
$$

where $\hat{A}_{i, \alpha}^{\dagger}:=\hat{a}_{2 i-1, \alpha}^{\dagger}+\lambda \hat{a}_{2 i, \alpha}^{\dagger}+\hat{a}_{2 i+1, \alpha}^{\dagger}$ determines the values of $t_{r, r^{\prime}}$ and $V_{r}$ in Eq. (1), and we assume $\lambda \in \mathbb{R} \backslash\{0\}$. Periodic boundary condition (PBC) has been imposed. $\hat{H}_{\text {saw }}$ is positive semidefinite, and it has two energy bands: a dispersive band with energy $\lambda^{2}+2+2 \cos k>0$ and a flat band with exactly zero energy. Every eigenstate of the flat band can be chosen to be localized on three sites (see Fig. 1):

$$
\hat{B}_{j, \alpha}^{\dagger}:=\frac{1}{\sqrt{\lambda^{2}+2}}\left(\hat{a}_{2 j, \alpha}^{\dagger}-\lambda \hat{a}_{2 j+1, \alpha}^{\dagger}+\hat{a}_{2 j+2, \alpha}^{\dagger}\right),
$$

where $\hat{B}_{j, \alpha}^{\dagger}$ creates a particle in a zero-energy eigenstate. In other words, $\hat{B}_{j, \alpha}^{\dagger}$ is a CLS creation operator. An experimental scheme for realizing an optical sawtooth chain has been proposed [58]. 
Note that lattices with a bottom flat band (and CLSs) widely exist; they can actually be constructed systematically, see Sec. III B.

From now on, the total particle number on the sawtooth chain is assumed to be the same as the number of unit cells $N$. For simplicity, we also assume translation symmetry: $g_{S, r} \equiv$ $g_{S}^{\mathrm{t}}$ for top sites $\left(r=\right.$ even) and $g_{S, r} \equiv g_{S}^{\mathrm{b}}$ for bottom sites $(r=$ odd). The phase diagram of the spin-1 BHMSC with respect to $\left(g_{0}^{\mathrm{t}}, g_{2}^{\mathrm{t}}, 1 / \lambda\right)$ is shown in Fig. 2(a).

For later purposes, we also introduce the $1 \mathrm{D}$ spin-1 BLBQ model with PBC, whose Hamiltonian is given by [36]

$$
\begin{aligned}
\hat{H}_{\mathrm{BLBQ}} & =\sum_{j=1}^{N}\left(\tilde{g}_{0} \hat{P}_{j, j+1}^{(0)}+\tilde{g}_{1} \hat{P}_{j, j+1}^{(1)}+\tilde{g}_{2} \hat{P}_{j, j+1}^{(2)}\right), \\
& =J \sum_{j=1}^{N}\left[\cos \theta\left(\hat{\boldsymbol{S}}_{j} \cdot \hat{\boldsymbol{S}}_{j+1}\right)+\sin \theta\left(\hat{\boldsymbol{S}}_{j} \cdot \hat{\boldsymbol{S}}_{j+1}\right)^{2}\right]+c,
\end{aligned}
$$

where $\hat{P}_{j, j+1}^{(F)}$ projects the state of two neighboring sites onto the state with total $\operatorname{spin} F=0,1,2$. Spin operators $\left\{\hat{\boldsymbol{S}}_{j}\right\}$ act on the spin-chain Hilbert space spanned by the $S^{z}$-basis $\left\{\left|\psi_{\alpha}\right\rangle:=\right.$ $\left.\left|\alpha_{1}, \alpha_{2}, \ldots, \alpha_{N}\right\rangle\right\}$. Parameters $(J \cos \theta, J \sin \theta, c)$ with $J>0$ linearly depend on $\left(\tilde{g}_{0}, \tilde{g}_{1}, \tilde{g}_{2}\right)$. This model is in the Haldane phase when $-\pi / 4<\theta<\pi / 4$, while it is in the critical phase when $\pi / 4 \leqslant \theta \leqslant \pi / 2$. At $\theta=\arctan (1 / 3)$ and $\pi / 2$, the model is particularly known as the AKLT model and the pure-biquadratic model $[59,60]$, respectively. For the 1D spin-1 AKLT model

$$
\hat{H}_{\mathrm{AKLT}}=\sum_{j=1}^{N} \tilde{g}_{2} \hat{P}_{j, j+1}^{(2)} \quad\left(\tilde{g}_{2}>0\right),
$$

the ground state $|\mathrm{VBS}\rangle$ under $\mathrm{PBC}$ is unique and can be exactly written as a matrix product state (MPS)

$$
|\mathrm{VBS}\rangle=\sum_{\alpha_{1}, \ldots, \alpha_{N}} \operatorname{Tr}\left(M^{\alpha_{1}} M^{\alpha_{2}} \cdots M^{\alpha_{N}}\right)\left|\psi_{\boldsymbol{\alpha}}\right\rangle,
$$

where $M^{ \pm 1}:=\mp \sqrt{2} \sigma^{ \pm}, M^{0}:=\sigma^{z}$, and $\sigma^{ \pm, z}$ are Pauli matrices. $|\mathrm{VBS}\rangle$ in Eq. (8) is known as the 1D spin-1 VBS state, which can be graphically represented as in Fig. 3(a). See Sec. III A for details.

We notice the analogy between the $s$-wave collision Hamiltonian $\hat{H}_{\text {int }}$ and the BLBQ Hamiltonian $\hat{H}_{\mathrm{BLBQ}}$. This will help us the find the exact ground state and the SPT phase in the spinful, itinerant, and short-range interacting bosonic systems.

\section{B. Exact ground states}

Since both $\hat{H}_{\text {saw }}$ and $\hat{H}_{\text {int }}$ are positive semidefinite, a zero-energy ground state of $\hat{H}$, if it exists, must satisfy (1) $\hat{H}_{\text {saw }}|\mathrm{GS}\rangle=0$ and (2) $\hat{H}_{\text {int }}|\mathrm{GS}\rangle=0$. In accordance with (1), there must be

$$
|\mathrm{GS}\rangle=\sum_{\sum_{j, \mu} n_{j, \mu}=N} x_{n}\left(\prod_{j=1}^{N} \prod_{\mu=-1}^{1}\left(\hat{B}_{j, \mu}^{\dagger}\right)^{n_{j, \mu}}\right)|\mathrm{vac}\rangle,
$$

where $x_{\boldsymbol{n}} \in \mathbb{C}, \boldsymbol{n}=\left(\ldots, n_{i, 1}, n_{i, 0}, n_{i,-1}, n_{i+1,1}, \ldots\right)$, and $|\mathrm{vac}\rangle$ is the vacuum state. Assume $g_{0}^{\mathrm{b}}, g_{2}^{\mathrm{b}}>0$, according to Eq. (3)
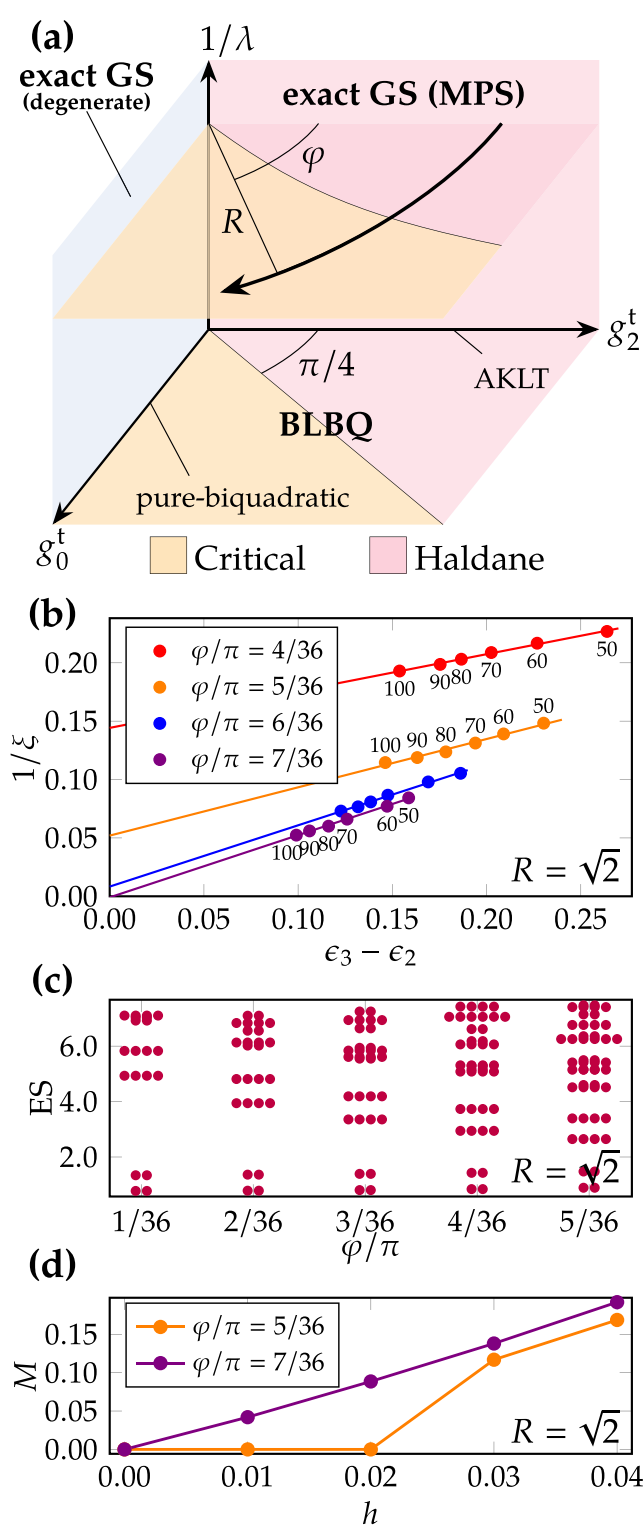

FIG. 2. (a) Schematic phase diagram of spin-1 bosons on a sawtooth chain (in the thermodynamic limit $N \rightarrow \infty$ ) in the parameter space $\left(g_{0}^{\mathrm{t}}, g_{2}^{\mathrm{t}}, 1 / \lambda\right)$. In the phase diagram we have assumed $g_{0}^{\mathrm{t}}, g_{2}^{\mathrm{t}}, \lambda \geqslant 0$ and $g_{0}^{\mathrm{b}}=g_{2}^{\mathrm{b}}=1 / \lambda^{2}$. In the $g_{0}^{\mathrm{t}}=0$ plane, $\hat{H}$ has an exact and unique ground state (GS) given in Eq. (19). In the $g_{2}^{\mathrm{t}}=$ 0 plane, the GS is massively degenerate, and the ferromagnetic states in Eq. (21) are exact ground states. Phase diagram in the $1 / \lambda \rightarrow 0$ plane, derived by perturbation theory, coincides with the phase diagram of the BLBQ model. Phase diagram in the $1 / \lambda=$ 1 plane is determined by the numerical calculation based on the VUMPS algorithm. In particular, numerical results along the curved arrow parameterized by $(\sqrt{2} \sin \varphi, \sqrt{2} \cos \varphi, 1)$ are shown in (b)-(d). (b) Scaling of the inverse correlation length $1 / \xi:=\epsilon_{2}$ with respect to $\epsilon_{3}-\epsilon_{2}$. Numbers near the data points denote the corresponding bond dimensions of each block; see Appendix E. We can see that a quantum phase transition occurs between $\varphi=6 \pi / 36$ and $7 \pi / 36$. (c) The whole entanglement spectrum (ES) in the Haldane phase region shows the even-fold degeneracy. For clarity, here we present only the lowest part of the ES. (d) Magnetization $M$ with respect to the applied magnetic field $h$ in the $z$-direction. In (c) and (d), the bond dimension of each block is 50 . 
(a)

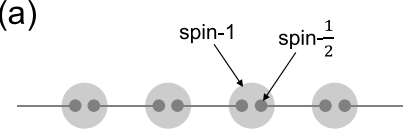

$$
\stackrel{\mathrm{a}}{\stackrel{b}{b}}=\frac{1}{\sqrt{2}}\left(\left|\uparrow_{\mathrm{a}}\right\rangle\left|\downarrow_{\mathrm{b}}\right\rangle-\left|\downarrow_{\mathrm{a}}\right\rangle\left|\uparrow_{\mathrm{b}}\right\rangle\right)
$$$$
=\frac{1}{2}(\rightarrow \cdots+\curvearrowleft)
$$

(b)

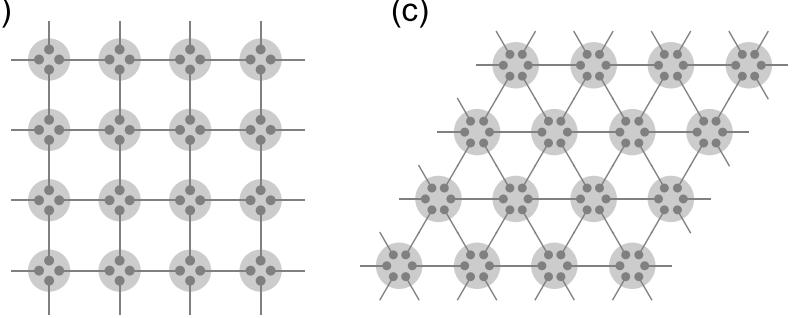

(d)

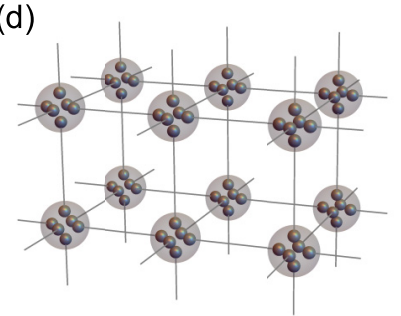

FIG. 3. Examples of bosonic VBS states. Each spin- $f$ is regarded as a composite state of $2 f$ spin-1/2's, and a spin singlet is formed between two spin-1/2's on neighboring sites. (a) 1D spin-1 VBS state, whose expression is given in Eq. (8). (b) 2D spin-2 VBS state on a square lattice, denoted as $\left|\mathrm{VBS}_{2, \square}\right\rangle$. (c) 2D spin-3 VBS state on a triangular lattice, denoted as $\left|\mathrm{VBS}_{3, \Delta}\right\rangle$. (d) $3 \mathrm{D}$ spin-3 VBS state on a cubic lattice, denoted as $\left|\mathrm{VBS}_{3, \mathbb{6}}\right\rangle$.

and the positive semidefiniteness of $\hat{P}_{r}^{(S)}$, one can conclude that

$$
\begin{aligned}
\hat{H}_{\text {int }}|\mathrm{GS}\rangle=0 & \\
& \Longleftrightarrow\left(g_{0}^{\mathrm{b}} \hat{P}_{2 j+1}^{(0)}+g_{2}^{\mathrm{b}} \hat{P}_{2 j+1}^{(2)}\right)|\mathrm{GS}\rangle=0, \forall j \\
& \Longleftrightarrow \hat{n}_{2 j+1}\left(\hat{n}_{2 j+1}-1\right)|\mathrm{GS}\rangle=0, \forall j \\
& \Longleftrightarrow \hat{a}_{2 j+1, \alpha} \hat{a}_{2 j+1, \beta}|\mathrm{GS}\rangle=0, \forall j, \alpha, \beta \\
& \Longrightarrow x_{\boldsymbol{n}}=0, \forall \boldsymbol{n} \text { s.t. } n_{j,+1}+n_{j, 0}+n_{j,-1}>1 .
\end{aligned}
$$

Equation (9) thus reduces to

$$
|\mathrm{GS}\rangle=\sum_{\boldsymbol{\beta}} y_{\boldsymbol{\beta}}|\boldsymbol{\beta}\rangle,
$$

where $y_{\boldsymbol{\beta}} \in \mathbb{C}, \boldsymbol{\beta}=\left(\beta_{1}, \ldots, \beta_{N}\right)$, and

$$
|\boldsymbol{\beta}\rangle:=\left(\prod_{j=1}^{N} \hat{B}_{j, \beta_{j}}^{\dagger}\right)|\mathrm{vac}\rangle
$$

A typical $|\boldsymbol{\beta}\rangle$ is illustrated in Fig. 1. Note that $|\boldsymbol{\beta}\rangle$ 's are linearly independent but not orthonormal because $K_{j j^{\prime}}:=$ $\left[\hat{B}_{j, \mu}, \hat{B}_{j^{\prime}, \mu}^{\dagger}\right] \neq \delta_{j j^{\prime}}$. We define the "dual operator" of $\hat{B}_{j, \mu}$ as [61]

$$
\hat{C}_{j, \mu}:=\sum_{j^{\prime}}\left(K^{-1}\right)_{j j^{\prime}} \hat{B}_{j^{\prime}, \mu}
$$

such that $\left[\hat{C}_{j, \mu}, \hat{B}_{j^{\prime}, \mu^{\prime}}^{\dagger}\right]=\delta_{j j^{\prime}} \delta_{\mu \mu^{\prime}}$. Further defining

$$
\langle\widetilde{\boldsymbol{\alpha}}|:=\langle\operatorname{vac}|\left(\prod_{j=1}^{N} \hat{C}_{j, \alpha_{j}}\right)
$$

such that $\langle\widetilde{\boldsymbol{\alpha}} \mid \boldsymbol{\beta}\rangle=\delta_{\boldsymbol{\alpha} \boldsymbol{\beta}}$, eigenequation $\hat{H}|\mathrm{GS}\rangle=0$ then implies the matrix equation $\sum_{\boldsymbol{\beta}}\langle\widetilde{\boldsymbol{\alpha}}|\hat{H}| \boldsymbol{\beta}\rangle_{y_{\beta}}=0$. Impressively, explicit calculation shows that

$$
\langle\widetilde{\boldsymbol{\alpha}}|\hat{H}| \boldsymbol{\beta}\rangle=\left\langle\widetilde{\boldsymbol{\alpha}}\left|\hat{H}_{\mathrm{int}}\right| \boldsymbol{\beta}\right\rangle=\left\langle\psi_{\boldsymbol{\alpha}}\left|\hat{H}_{\mathrm{BLBQ}}\right| \psi_{\boldsymbol{\beta}}\right\rangle,
$$

provided that we take $\tilde{g}_{1}=0$ and $\tilde{g}_{S}=2 g_{S}^{\mathrm{t}} d /\left(\lambda^{2}+2\right)$ in Eq. (6), where $d>0$ is a coefficient depending on the inverse matrix $K^{-1}$ (matrix $K$ is always invertable). Equation (15) indicates that there is a one-to-one correspondence between the zero-energy states of $\hat{H}$ and $\hat{H}_{\mathrm{BLBQ}}$. Note that such correspondence does not hold for eigenstates with nonzero energy, because $\hat{P}_{r=\text { odd }}^{(S)}|\boldsymbol{\beta}\rangle=0$ implies that nonzero-energy eigenstates cannot be purely spanned by $\{|\boldsymbol{\beta}\rangle\}$. It is known that in the following two cases, $\hat{H}_{\mathrm{BLBQ}}$ possesses zero-energy ground states: (1) AKLT point $\left(\tilde{g}_{0}=\tilde{g}_{1}=0, \tilde{g}_{2}>0\right.$ ) and (2) pure-biquadratic point $\left(\tilde{g}_{1}=\tilde{g}_{2}=0, \tilde{g}_{0}>0\right.$ ).

Case (1) maps to $g_{0}^{\mathrm{t}}=0$ and $g_{2}^{\mathrm{t}}>0$ for $\hat{H}$. In this case, the ground state of $\hat{H}$ is unique, which follows from the uniqueness of the ground state of the AKLT model [2,3]. Despite the fact that the $|\mathrm{GS}\rangle$ in Eq. (11) is not represented in an orthonormal basis, the coefficient $y_{\beta}$ is identical to that of the 1D VBS state in Eq. (8):

$$
y_{\boldsymbol{\beta}}=\operatorname{Tr}\left(M^{\beta_{1}} M^{\beta_{2}} \cdots M^{\beta_{N}}\right) .
$$

Further expanding $\hat{B}_{j, \beta_{j}}^{\dagger}$ in terms of $\hat{a}^{\dagger}$ 's, we can see that $|\mathrm{GS}\rangle$ is a superposition of states of the form

$$
(-\lambda)^{b} \sum_{\beta} \operatorname{Tr}\left(M^{\beta_{1}} M^{\beta_{2}} \cdots M^{\beta_{N}}\right) \hat{a}_{r_{1}, \beta_{1}}^{\dagger} \hat{a}_{r_{2}, \beta_{2}}^{\dagger} \ldots \hat{a}_{r_{N}, \beta_{N}}^{\dagger},
$$

where the integer $b$ depends on $\left\{r_{1}, \ldots, r_{N}\right\}$. In Eq. (17), we note that as long as two particles occupy the same top site $\ell$, there is the identity

$$
\sum_{\beta_{j}, \beta_{j+1}} M^{\beta_{j}} M^{\beta_{j+1}} \hat{a}_{\ell, \beta_{j}}^{\dagger} \hat{a}_{\ell, \beta_{j+1}}^{\dagger}=\sqrt{6} \hat{b}_{\ell}^{\dagger} I_{2},
$$

where $\ell=2 j$ or $2 j+2$ and $I_{2}$ is a 2 -by-2 identity matrix. Equation (18) implies that Eq. (17) has "hidden VBS order," i.e., if we ignore all the vacant sites and sites occupied by a spin singlet, the remaining bosons form a perfect VBS state; see Fig. 1. This enables us to express $|\mathrm{GS}\rangle$ in an orthonormal Fock basis as

$$
|\mathrm{GS}\rangle=\sum_{\tau_{1}, \ldots, \tau_{2 N}=-1}^{3} \operatorname{Tr}\left(\prod_{j=1}^{N}\left(F^{\tau_{2 j-1}} E^{\tau_{2 j}}\right)\right)\left(\prod_{r=1}^{2 N} \hat{d}_{r, \tau_{r}}^{\dagger}\right)|\mathrm{vac}\rangle,
$$

where $\hat{d}_{r, \tau}^{\dagger}:=\hat{a}_{r, \tau}^{\dagger}$ for $\tau=-1,0,1$, while $\hat{d}_{r, 2}^{\dagger}:=\hat{b}_{r}^{\dagger}$ and $\hat{d}_{r, 3}^{\dagger}:=1$, and

$$
\begin{aligned}
& \sum_{\tau=-1}^{3} F^{\tau} \hat{d}_{r, \tau}^{\dagger}=\frac{1}{\sqrt{\lambda^{2}+2}}\left(\begin{array}{cc}
I_{2} & -\lambda \sum_{\alpha} M^{\alpha} \hat{a}_{r, \alpha}^{\dagger} \\
0 & I_{2}
\end{array}\right), \\
& \sum_{\tau=-1}^{3} E^{\tau} \hat{d}_{r, \tau}^{\dagger}=\left(\begin{array}{cc}
\sum_{\alpha} M^{\alpha} \hat{a}_{r, \alpha}^{\dagger} & \sqrt{6} \hat{b}_{r}^{\dagger} I_{2} \\
I_{2} & \sum_{\alpha} M^{\alpha} \hat{a}_{r, \alpha}^{\dagger}
\end{array}\right) .
\end{aligned}
$$


Matrices $F^{\tau}$ and $E^{\tau}$ are determined from Eq. (20); the matrix product state (MPS) in Eq. (19) is injective [62]. Using Eq. (20), one can easily see that the ground state in Eq. (19) is indeed a superposition of 1D VBS states decorated with two-body singlets and/or vacant sites.

Case (2) maps to $g_{2}^{\mathrm{t}}=0$ and $g_{0}^{\mathrm{t}}>0$ for $\hat{H}$. It is obvious that the ferromagnetic states

$$
\left(\sum_{r=1}^{2 N} \hat{S}_{r}^{-}\right)^{k}\left(\prod_{j=1}^{N} \hat{B}_{j, 1}^{\dagger}\right)|\mathrm{vac}\rangle, \quad k=0,1, \ldots, 2 N
$$

with total spin $S_{\text {tot }}=N$ are exact ground states of $\hat{H}$. The spin1 pure-biquadratic chain $\hat{H}_{\mathrm{PB}}=\sum_{j=1}^{N} \tilde{g}_{0} \hat{P}_{j, j+1}^{(0)}\left(\right.$ with $\tilde{g}_{0}>0$ ) is integrable, and there are numerous ground states with $S_{\text {tot }}$ ranging from 0 to $N-1$ that are degenerate with $\left(\sum_{j} \hat{S}_{j}^{-}\right)^{k}\left|\psi_{(1,1, \ldots, 1)}\right\rangle[59,60,63,64]$. The absence of a ferromagnetic phase in Fig. 2(a) can thus be understood from such degeneracy: after adding interaction $\sum_{r=\text { even }} g_{2}^{\mathrm{t}} \hat{P}_{r}^{(2)}$ (with $g_{2}^{\mathrm{t}}>0$ ) that disfavors the ferromagnetic states, states with smaller $S_{\text {tot }}$ are picked up as the ground states.

\section{The Haldane phase}

In this section we investigate the properties of the MPS $|\mathrm{GS}\rangle$. Let $G$ be the symmetry group of $\hat{H}$ and $\hat{U}(q)$ be a symmetry operation (on the Hilbert space) corresponding to the group element $q \in G$, i.e., $[\hat{H}, \hat{U}(q)]=0$. Subjected to $q$, the unique ground state transforms as $|\mathrm{GS}\rangle \rightarrow \hat{U}(q)|\mathrm{GS}\rangle$, while the matrices in Eq. (19) transform as [65]

$$
F^{\tau_{2 j-1}} E^{\tau_{2 j}} \rightarrow e^{i \phi_{q}} u_{q}^{\dagger} F^{\tau_{2 j-1}} E^{\tau_{2 j}} u_{q},
$$

where $\left\{u_{q}\right\}_{q \in G}$ are unitary matrices which are used to classify the 1D SPT phases $[4,7,66]$.

The group $\mathbb{Z}_{2} \times \mathbb{Z}_{2}=\{1, \hat{U}(x), \hat{U}(y), \hat{U}(z)\}$ is a symmetry group of $\hat{H}$, where $\hat{U}(\delta):=\exp \left(-i \pi \sum_{r} \hat{S}_{r}^{\delta}\right)$ is the spin rotation about the $\delta=x, y, z$-axis. The Hamiltonian is also invariant under time-reversal $\hat{U}(\mathrm{TR}):=$ $\hat{U}(y) \hat{K}$ (where $\hat{K}$ is a complex conjugation operator), space inversion $\hat{U}(\mathcal{I})$, spin rotation together with inversion $\hat{U}(z \mathcal{I}):=\hat{U}(z) \hat{U}(\mathcal{I})$, and pseudospin rotation together inversion $\hat{U}(n \mathcal{I}):=\exp \left[-i \pi \sum_{r}\left(\hat{n}_{r}-1\right)\right] \hat{U}(\mathcal{I})$.

For $\hat{U}(\delta)$ and $\hat{U}(\mathrm{TR})$, we can define their respective topological indices using the corresponding unitary matrices in Eq. (22) as $\mathcal{Q}_{\mathbb{Z}_{2} \times \mathbb{Z}_{2}}:=\operatorname{Tr}\left(u_{x} u_{z} u_{x}^{\dagger} u_{z}^{\dagger}\right) / \chi$ and $\mathcal{Q}_{\mathrm{TR}}:=$ $\operatorname{Tr}\left(u_{\mathrm{TR}} u_{\mathrm{TR}}^{*}\right) / \chi$, where $\chi$ is the bond dimension of the MPS [67]. It is known that $\mathcal{Q}_{\mathbb{Z}_{2} \times \mathbb{Z}_{2}}$ equals -1 for the Haldane phase protected by $\mathbb{Z}_{2} \times \mathbb{Z}_{2}$ symmetry while 1 for the trivial phase, similarly for $\mathcal{Q}_{\mathrm{TR}}$ [4]. When $0<|\lambda|<\infty$, the system has inversion symmetry with respect to every lattice site. However, the site-centered inversion symmetry cannot protect SPT phases. The groups $\{1, \hat{U}(\mathcal{I})\},\{1, \hat{U}(z \mathcal{I})\}$, and $\{1, \hat{U}(n \mathcal{I})\}$ can protect SPT phases only when $\hat{U}(\mathcal{I})$ is a bond-centered inversion; see also Ref. [68] and Appendix A of Ref. [69]. When the bond-centered inversion symmetry is present, we can similarly define $\mathcal{Q}_{\mathcal{I}}:=\operatorname{Tr}\left(u_{\mathcal{I}} u_{\mathcal{I}}^{*}\right) / \chi, \mathcal{Q}_{z \mathcal{I}}:=\operatorname{Tr}\left(u_{z \mathcal{I}} u_{z \mathcal{I}}^{*}\right) / \chi$, and $\mathcal{Q}_{n \mathcal{I}}:=\operatorname{Tr}\left(u_{n \mathcal{I}} u_{n \mathcal{I}}^{*}\right) / \chi$, which are quantized to +1 and -1 for trivial and Haldane phases, respectively $[4,5,67]$. The state $|\mathrm{GS}\rangle$ at $\lambda=0$ and $|\lambda| \rightarrow \infty$ has bond-centered inversion symmetry. At $|\lambda| \rightarrow \infty,|\mathrm{GS}\rangle$ reduces to Eq. (8). At $\lambda=0$, although $\left|G S_{\lambda=0}\right\rangle$ is not the unique ground state of $\left.\hat{H}\right|_{\lambda=0}$, one can (in principle) always find a parent Hamiltonian that has $\left|\mathrm{GS}_{\lambda=0}\right\rangle$ as the unique ground state [70], and hence the state itself is still worth studying. Actually, it turns out that $\left|\mathrm{GS}_{\lambda=0}\right\rangle$ can be viewed as a spinful generalization of the Haldane insulator state in spinless bosons; see Appendix A. Table I summarizes the unitary matrices $\left\{u_{q}\right\}$ with respect to different symmetry operations on $|\mathrm{GS}\rangle$. It is then clear that the Haldane phase of $\left|G_{0<|\lambda|<\infty}\right\rangle$ is protected by $\mathbb{Z}_{2} \times \mathbb{Z}_{2}$ symmetry or time-reversal symmetry. Interestingly, when the inversion symmetry is involved, $\left|\mathrm{GS}_{\lambda=0}\right\rangle$ and $\left|\mathrm{GS}_{|\lambda| \rightarrow \infty}\right\rangle$ can be in different phases. This difference originates from the charge fluctuations in $\left|\mathrm{GS}_{\lambda=0}\right\rangle$. We claim that in general, charge fluctuations can play a nontrivial role in the SPT orders protected by crystalline symmetries; see Sec. III C for details.

Using the exact MPS in Eq. (19) and Eq. (20), various quantities that characterize the Haldane phase can be calculated analytically. For example, the spin string order parameter $\mathcal{O}^{\delta}:=-\lim _{L \rightarrow \infty} \lim _{N \rightarrow \infty} \frac{1}{\langle\mathrm{GS} \mid \mathrm{GS}\rangle}\langle\mathrm{GS}|\left(\hat{S}_{r}^{\delta}+\hat{S}_{r+1}^{\delta}\right)$ $\exp \left[i \pi \sum_{k=r+2}^{r+2 L-1} \hat{S}_{k}^{\delta}\right]\left(\hat{S}_{r+2 L}^{\delta}+\hat{S}_{r+2 L+1}^{\delta}\right)|\mathrm{GS}\rangle$ and the charge string order parameter $\mathcal{C}:=-\lim _{L \rightarrow \infty} \lim _{N \rightarrow \infty} \frac{1}{\langle\mathrm{GS} \mid \mathrm{GS}\rangle}\langle\mathrm{GS}|$ $\left(\hat{n}_{r}+\hat{n}_{r+1}-1\right) \exp \left\{i \pi\left[\sum_{k=r+2}^{r+2 L-1} \hat{n}_{k}-(L-1)\right]\right\}\left(\hat{n}_{r+2 L}+\right.$ $\left.\hat{n}_{r+2 L+1}-1\right)|\mathrm{GS}\rangle$ are found to be

$$
\begin{aligned}
\mathcal{O}^{\delta} & =\frac{16\left[9 \lambda^{6}+(5 Q+48) \lambda^{2}+3(Q+11) \lambda^{4}+24\right]^{2}}{Q^{2}\left(3 \lambda^{2}+Q+6\right)^{2}\left(Q+3 \lambda^{2}\right)^{2}}, \\
\mathcal{C} & =\frac{24\left(3 \lambda^{2}+2\right)^{2}}{Q^{2}\left[3 \lambda^{4}+(Q+12) \lambda^{2}+2(Q+5)\right]},
\end{aligned}
$$

where $Q:=\sqrt{9 \lambda^{4}+36 \lambda^{2}+24}$. It can be shown that both string order parameters are nonzero: $4 /(\sqrt{6}+3)^{2}<\mathcal{O}^{\delta}<$ $4 / 9$ and $0<\mathcal{C}<0.207$. For the open boundary condition (OBC), we can show the existence of both spin and charge edge states; see Appendix B. It is known that the (seemingly unrelated) spin string order and edge state are unified in the context of hidden $\mathbb{Z}_{2} \times \mathbb{Z}_{2}$ symmetry breaking. In spin chains, this can be seen with the Kennedy-Tasaki transformation [5,7-9]. In spin- $f$ itinerant systems ( $f=$ integer), the Kennedy-Tasaki transformation is also applicable; see Appendix C.

The "hidden VBS order" is a unique feature for the Haldane phase in systems with both spin and charge fluctuations. It is closely related to both string order parameters $\mathcal{O}^{\delta}$ and $\mathcal{C}$. Since vacant and doubly occupied sites have zero spin, the "hidden VBS order" immediately implies the hidden antiferromagnetic order measured by $\mathcal{O}^{\delta}$. On the other hand, $\mathcal{C}$ measures to what extent the VBS states are diluted in the background of vacant and doubly occupied sites.

In the presence of both translation symmetry and $\mathbb{Z}_{2} \times \mathbb{Z}_{2}$ symmetry, four distinct SPT phases can exist [71], and one of them is represented by $|\mathrm{GS}\rangle$. The other three can be realized by unitary transformations of $\hat{H}_{\text {hop }}$; see Appendix D for details.

\section{Perturbation theory}

Beyond the cases where the ground state is exactly solvable, the phase of $\hat{H}$ can still be determined analytically when $|\lambda|$ is large enough; see Fig. 2(a). In the limit $|\lambda| \rightarrow \infty$, if we assume $g_{0}^{\mathrm{b}}$ and $g_{2}^{\mathrm{b}}$ are around the magnitude of $\lambda^{2}$, the 
TABLE I. Unitary matrices in Eq. (22) with respect to various symmetry operations on $|\mathrm{GS}\rangle$. In accordance with the values of $\mathcal{Q}_{\mathbb{Z}_{2} \times \mathbb{Z}_{2}}$, $\mathcal{Q}_{\mathrm{TR}}, \mathcal{Q}_{\mathcal{I}}, \mathcal{Q}_{z \mathcal{I}}$, and $\mathcal{Q}_{n \mathcal{I}}$, the two boldfaced matrices denote trivial phases, while the other matrices denote the SPT phase. N/A means the symmetry group cannot give an SPT/trivial classification.

\begin{tabular}{|c|c|c|c|c|c|}
\hline & $\hat{U}(\delta)(\delta=x, y, z)$ & $\hat{U}(\mathrm{TR})$ & $\hat{U}(\mathcal{I})$ & $\hat{U}(z \mathcal{I})$ & $\hat{U}(n \mathcal{I})$ \\
\hline$\lambda=0$ & $u_{\delta}=\left(\begin{array}{cc}\sigma^{\delta} & 0 \\
0 & \sigma^{\delta}\end{array}\right)$ & $u_{\mathrm{TR}}=\left(\begin{array}{cc}\sigma^{y} & 0 \\
0 & \sigma^{y}\end{array}\right)$ & $u_{\mathcal{I}}=\left(\begin{array}{cc}0 & -\sigma^{y} \\
\sigma^{y} & 0\end{array}\right)$ & $u_{z \mathcal{I}}=\left(\begin{array}{cc}0 & -\sigma^{x} \\
\sigma^{x} & 0\end{array}\right)$ & $u_{n \mathcal{I}}=\left(\begin{array}{c}0 \\
\sigma^{y}\end{array}\right.$ \\
\hline $0<|\lambda|<\infty$ & $u_{\delta}=\left(\begin{array}{cc}\sigma^{\delta} & 0 \\
0 & \sigma^{\delta}\end{array}\right)$ & $u_{\mathrm{TR}}=\left(\begin{array}{cc}\sigma^{y} & 0 \\
0 & \sigma^{y}\end{array}\right)$ & N/A & N/A & $\mathrm{N} / \mathrm{A}$ \\
\hline$|\lambda| \rightarrow \infty$ & $u_{\delta}=\sigma^{\delta}$ & $u_{\mathrm{TR}}=\sigma^{y}$ & $u_{\mathcal{I}}=\sigma^{y}$ & $\boldsymbol{u}_{z \mathcal{I}}=\sigma^{\boldsymbol{x}}$ & $u_{n \mathcal{I}}=\sigma^{y}$ \\
\hline
\end{tabular}

unperturbed ground state will have each bottom site occupied by exactly one particle. In this case, perturbation theory tells us that the low-energy effective Hamiltonian of $\hat{H}$ is given by $\hat{H}_{\text {BLBQ }}$ in Eq. (6) with $J \propto \lambda^{-2}$ and

$$
\theta=\arctan \left(\frac{1}{3} \times \frac{3 g_{0}^{\mathrm{t}}+4 g_{0}^{\mathrm{t}} \lambda^{2} / g_{2}^{\mathrm{t}}+2 \lambda^{2}}{g_{0}^{\mathrm{t}}+2 \lambda^{2}}\right) \geqslant \arctan \frac{1}{3},
$$

where $J$ and $\theta$ are independent of $g_{S}^{\mathrm{b}}$. From Eq. (24), we know that the effective model is in the Haldane phase when $0 \leqslant g_{0}^{\mathrm{t}}<g_{2}^{\mathrm{t}}$ while in the critical phase when $0<g_{2}^{\mathrm{t}} \leqslant g_{0}^{\mathrm{t}}$. In particular, $g_{0}^{\mathrm{t}}=0$ and $g_{2}^{\mathrm{t}}=0$ corresponds to the AKLT and pure-quadratic point, respectively.

\section{E. Numerical analysis}

Beyond the three special planes in Fig. 2(a) where either exact ground states can be found or perturbation theory works, the phase diagram of the spin-1 BHMSC can in general be determined by numerical calculations. In the thermodynamic limit $N \rightarrow \infty$, we find the phase diagram in the $\lambda=1$ plane in Fig. 2(a) with the VUMPS algorithm [50,51]. Due to the fact that the total number of particles and unit cells are the same, matrices in the MPS ansatz used in the algorithm are assumed to be block-banded [72]. Also, the maximum particle number on each site is truncated to three. See Appendix E for details of the MPS ansatz. Let $\epsilon_{i}:=-\ln \left|\lambda_{i}\right|$, where $\lambda_{i}$ is the $i$ th largest absolute eigenvalue of the transfer matrix, and $\left|\lambda_{1}\right|$ is normalized to 1 . When the bond dimension $\chi$ is extrapolated to infinity, the correlation length $\xi:=1 / \epsilon_{2}$ diverges for gapless phases while it converges to a finite value for gapped phases. This fact is known to be well reflected in the scaling relation of $1 / \xi(\chi)$ with respect to $\epsilon_{3}(\chi)-\epsilon_{2}(\chi)$ [73,74], as shown in Fig. 2(b). In the region of the gapped phase in Fig. 2(a), we find that $\mathcal{Q}_{\mathbb{Z}_{2} \times \mathbb{Z}_{2}}=\mathcal{Q}_{\mathrm{TR}}=-1$, which suggests that the gapped phase is the Haldane phase. The Haldane phase is characterized by an even-fold degenerate entanglement spectrum [4]; see Fig. 2(c). The ground state magnetization $M:=\lim _{N \rightarrow \infty}\left\langle\sum_{r=1}^{2 N} \hat{S}_{r}^{z}\right\rangle / N$ is calculated after adding $-h \sum_{r} \hat{S}_{r}^{z}$ to $\hat{H}$, where $h$ is the magnetic field; see Fig. 2(d). In the gapless region, $M$ grows almost linearly with $h$, which suggests that the gapless phase is the critical phase [75]. In the Haldane phase region, however, $M$ is expected to exhibit a zero plateau for small $h$ [75], which is indeed the case as in Fig. 2(d). Note that the phase boundary in the $\lambda=1$ plane is curved; see Appendix E for numerical evidence.

\section{F. Short summary for the spin-1 bosons on a sawtooth chain}

To demonstrate how the Haldane phase emerges in shortrange interacting spinful bosons loaded on lattices with a bottom flat band, we have used the spin-1 BHMSC as an example. We show that this system has some deep connections with the BLBQ model. In particular, in a special case, by an exact mapping to the ground state of the AKLT model, we obtain the exact and unique ground state of the spin-1 BHMSC. This exact ground state turns out to serve as a representative state of the Haldane phase. The phase diagram of this model is obtained by perturbation theory and numerical calculations based on the VUMPS algorithm, and we find that the Haldane phase exists in a rather wide parameter region.

We expect that, even if the bottom band is not perfectly flat, the nature of the many-body ground states should remain unchanged as long as the interaction strength is sufficiently strong. Such robustness of the ground states has been rigorously proved in some classes of Hubbard models with a nearly flat band [7,57].

\section{GENERAL THEORY}

The sawtooth chain is not special in the sense that there are many other lattices possessing a bottom flat band, it is thus natural to expect that the SPT phases can be realized with spinful bosons loaded on these lattices. Our approach in the previous section can be generalized. In this section, we present a general theory for the SPT phases of spin- $f$ bosons with a bottom flat band. We first show in Sec. III A that the AKLT model and VBS state can be generalized to higher spins and higher dimensional lattices. Let $\left|\mathrm{VBS}_{f, X^{\prime}}\right\rangle$ be the exact and unique ground state of the spin- $f$ AKLT model defined on a lattice $X^{\prime}$. On the other hand, bottom-flat-band lattices can be constructed systematically. Let $\left|\mathrm{GS}_{f, X}\right\rangle$ be the ground state of $N$ spin- $f$ bosons on a bottom-flat-band lattice $X$ with $N$ unit cells. In Sec. III B, we show that with fine-tuned parameters, $\left|\mathrm{GS}_{f, X}\right\rangle$ can be exactly mapped to $\left|\mathrm{VBS}_{f, X^{\prime}}\right\rangle$, provided that $f, X$, and $X^{\prime}$ satisfy a certain relation. This means that $\left|\mathrm{GS}_{f, X}\right\rangle$ is the exact and unique ground state of the itinerant spin- $f$ model. In Sec. III C, with various $f$ and $X$, we classify the quantum phases of $\left|\mathrm{GS}_{f, X}\right\rangle$ 's from the viewpoint of SPT orders. In particular, we find that in terms of crystalline symmetries, not only spin fluctuations but also charge fluctuations in $\left|\mathrm{GS}_{f, X}\right\rangle$ determine its symmetry-protected phase. 
TABLE II. Some alkali-metal isotopes and their hyperfine spins $f$. These isotopes are stable or long lived (compared to typical experimental cycle time of around $10 \mathrm{~s}$ ) at least in lower hyperfine levels [33].

\begin{tabular}{lc}
\hline \hline Atom & $f$ \\
\hline${ }^{7} \mathrm{Li},{ }^{23} \mathrm{Na},{ }^{41} \mathrm{~K},{ }^{87} \mathrm{Rb}$ & 1,2 \\
${ }^{25} \mathrm{Na},{ }^{79,83} \mathrm{Rb},{ }^{131} \mathrm{Cs}$ & 2,3 \\
${ }^{135,137,139,141} \mathrm{Cs}$ & 3,4 \\
${ }^{119} \mathrm{Cs},{ }^{207,209,211,213} \mathrm{Fr}$ & 4,5 \\
\hline \hline
\end{tabular}

\section{A. Generalized AKLT models and VBS states}

It is known that VBS states can be constructed on any lattice in any dimensions $[3,76,77]$. In this article, we consider only bosonic spin- $f$ VBS states $\left(f=\right.$ integer). Let $X^{\prime}=$ $\left(\Lambda_{X^{\prime}}, \mathscr{B}_{X^{\prime}}\right)$ be a lattice (graph) where $\Lambda_{X^{\prime}}$ is the set of sites (vertices) and $\mathscr{B}_{X^{\prime}}$ is the set of bonds (edges). A bond is defined by two sites $\left\{\boldsymbol{j}, \boldsymbol{j}^{\prime}\right\}$ with $\boldsymbol{j}, \boldsymbol{j}^{\prime} \in \Lambda_{X^{\prime}}$. We assume that every site in $X^{\prime}$ is directly connected to $2 f$ other sites, i.e., $\left|\left\{\boldsymbol{j}^{\prime} \in \Lambda_{X^{\prime}} \mid\left\{\boldsymbol{j}, \boldsymbol{j}^{\prime}\right\} \in \mathscr{B}_{X^{\prime}}\right\}\right|=2 f, \forall \boldsymbol{j}$. (In other words, $X^{\prime}$ is a regular graph of degree $2 f$.) When there is a spin- $f$ degree of freedom (DOF) residing in every site of $X^{\prime}$, an AKLT-type quantum spin model can be defined on $X^{\prime}$ as

$$
\hat{H}_{\mathrm{AKLT}}^{f, X^{\prime}}:=\tilde{g}_{2 f} \sum_{\left\{\boldsymbol{j}, \boldsymbol{j}^{\prime}\right\} \in \mathscr{B}_{X^{\prime}}} \hat{P}_{\boldsymbol{j}, \boldsymbol{j}^{\prime}}^{(2 f)} \quad\left(\tilde{g}_{2 f}>0\right),
$$

where the operator $\hat{P}_{j, j^{\prime}}^{(2 f)}$ projects the state of two spin- $f$ 's on two sites $\boldsymbol{j}, \boldsymbol{j}^{\prime}$ onto the state with total spin $2 f$.

It has been proved that when $\left|\Lambda_{X^{\prime}}\right|=N<\infty, \hat{H}_{\mathrm{AKLT}}^{f, X^{\prime}}$ has an exact and unique ground state $[7,78]$, known as a VBS state:

$$
\left|\mathrm{VBS}_{f, X^{\prime}}\right\rangle=\sum_{\alpha_{1}, \ldots, \alpha_{N}=-f}^{f} S_{\alpha_{1}, \ldots, \alpha_{N}}\left|\psi_{\alpha_{1}, \ldots, \alpha_{N}}\right\rangle,
$$

where $\left\{\left|\psi_{\alpha_{1}, \alpha_{2}, \ldots, \alpha_{N}}\right\rangle\right\}$ is the spin $S^{z}$-basis and the coefficient $S_{\alpha_{1}, \ldots, \alpha_{N}}$ encodes short-range entanglement between the spins. When $X^{\prime}$ is the simple $1 \mathrm{D}$ linear chain with $f=1, \hat{H}_{\mathrm{AKLT}}^{f, X^{\prime}}$ reduces to Eq. (7), and its ground state is the 1D spin-1 VBS state in Eq. (8) and $S_{\alpha_{1}, \ldots, \alpha_{N}}=y_{\boldsymbol{\alpha}}$ in Eq. (16). The structure of this VBS state can be understood as follows: as shown in Fig. 3(a), each spin-1 is viewed as a composite state of two spin-1/2's, and a pair of spin-1/2's on two neighboring sites forms a spin singlet. For $\hat{H}_{\mathrm{AKLT}}^{f, X^{\prime}}$ on a general $X^{\prime}$, the ground state $\left|\mathrm{VBS}_{f, X^{\prime}}\right\rangle$ can be constructed in the same manner: each spin- $f$ is regarded as a composite state of $2 f$ spin-1/2's, and a singlet is formed between two spin-1/2's in every bond $\left\{\boldsymbol{j}, \boldsymbol{j}^{\prime}\right\} \in \mathscr{B}_{X^{\prime}}[3,76]$. Some other graph representations of VBS states in two and three dimensions are given in Figs. 3(b)-3(d).

\section{B. Ground states of spin- $f$ bosons with a bottom flat band}

Many kinds of atoms carry integer spins, among which alkali-metal atoms are often used in experiments [33]. Alkalimetal atoms have two hyperfine levels, and each level carries integer spin $f$; see Table II. Due to the hyperfine interaction, the level with smaller $f$ has lower energy. Therefore,

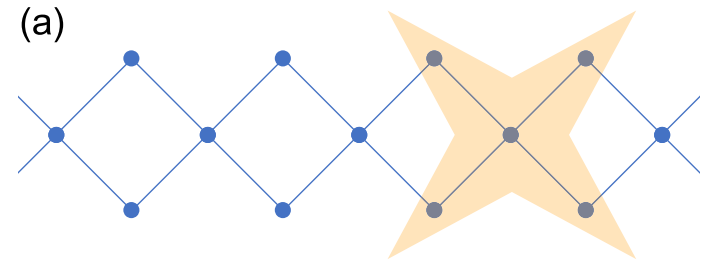

(b)

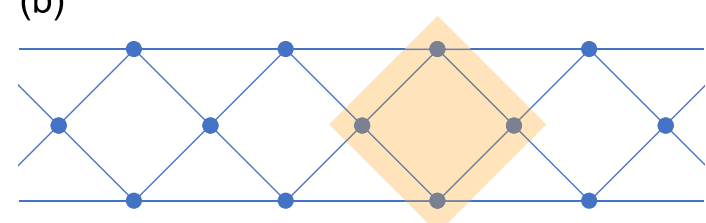

(c)

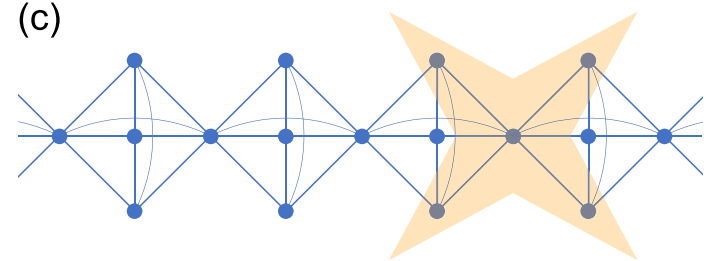

FIG. 4. Other examples of 1D lattices that can be used to construct nontrivial ground states with spin-1 bosons. Allowed hopping process between two sites is illustrated by a bond. (a) Diamond chain. A $\pi$ flux threads each plaquette [88]. A CLS covers five sites, as denoted by the four-pointed star. (b) Kagome ladder, an example of the line graph construction $[77,80]$. A CLS is denoted by the square. (c) Pyramid chain, an example of the cell construction [7]. A CLS is denoted by the four-pointed star.

alkali-metal atoms stay in lower hyperfine level when they are optically trapped without external pumping. For example, as shown in Table II, ${ }^{87} \mathrm{Rb}$ atoms are often regarded as spin-1 bosons, while they can indeed be spin- 2 bosons if one pumps them into the $f=2$ hyperfine level [33].

We have seen that the single-particle CLSs play a crucial role in constrcting the many-body ground state. The CLSs exist not only in the sawtooth chain but also in all the finiterange hopping lattices possessing a flat band [48,49]. In fact, there are various systematic approaches to construct flat-band lattices [7,48,55,56,79-86], among which Tasaki's cell construction [55,56] and Mielke's line graph construction [79-81] always yield a bottom flat band [87]. These systematic constructions generate infinitely many kinds of lattices in $d \geqslant 1$ dimensions, such as those shown in Fig. 4 for $d=1$ and Fig. 5 for $d=2,3$.

Let $X=\left(\Lambda_{X}, \mathscr{B}_{X}\right)$ be a bottom-flat-band lattice where $\Lambda_{X}$ is the set of sites and $\mathscr{B}_{X}$ is the set of bonds. A bond is defined by two sites $\left\{\boldsymbol{r}, \boldsymbol{r}^{\prime}\right\}$ with $\boldsymbol{r}, \boldsymbol{r}^{\prime} \in \Lambda_{X}$. Let $\hat{H}_{\text {hop }}^{f, X}$ be a single-body Hamiltonian for spin- $f$ bosons on $X$ :

$$
\hat{H}_{\text {hop }}^{f, X}=-\sum_{\left\{\boldsymbol{r}, \boldsymbol{r}^{\prime}\right\} \in \mathscr{B}_{X}} \sum_{\alpha=-f}^{f} t_{\boldsymbol{r}, \boldsymbol{r}^{\prime}} \hat{a}_{\boldsymbol{r}, \alpha}^{\dagger} \hat{a}_{\boldsymbol{r}^{\prime}, \alpha}+\sum_{\boldsymbol{r} \in \Lambda_{X}} V_{\boldsymbol{r}} \hat{n}_{\boldsymbol{r}},
$$

where $\hat{a}_{\boldsymbol{r}, \alpha}^{\dagger}$ creates a boson with magnetic sublevel $\alpha$ at site $\boldsymbol{r}$. Let $N$ be the total number of unit cells in $X$. The assumption that $X$ has a bottom flat band means that the 
$X$

(a)
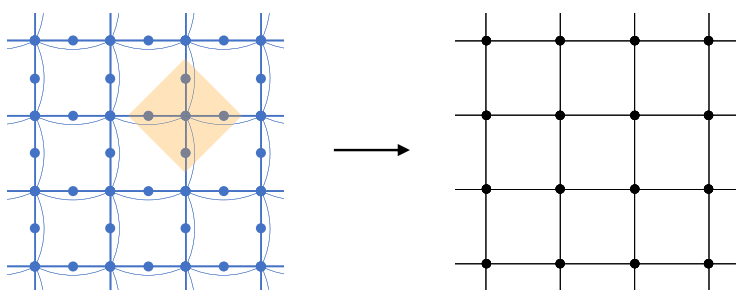

(b)
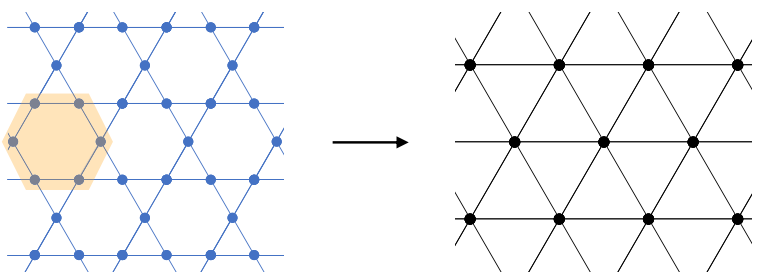

(c)

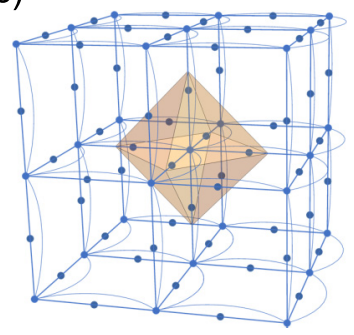

$X^{\prime}$

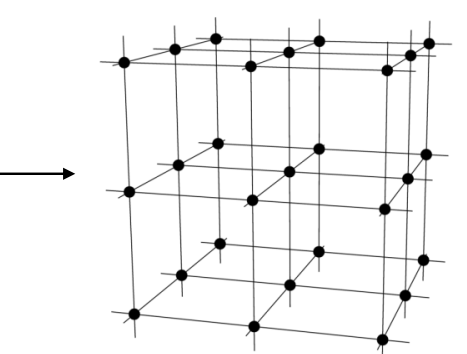

FIG. 5. Examples of higher dimensional lattices $X$ with a bottom flat band (left column) and their corresponding lattices $X^{\prime}$ where the AKLT models are defined (right column). In $X$, allowed hopping processes are illustrated by bonds. In $X^{\prime}$, a bond represents interaction between two spins. (a) 2D Tasaki lattice (left), an example of the cell construction [55,56]. A CLS is localized on five sites, as pictured by the square. All the CLSs are related to each other by lattice translation vectors. Every CLS overlaps with four other CLSs, thus $f$ should be $4 / 2=2$. The corresponding AKLT model lives on a square lattice (right). We symbolize the $2 \mathrm{D}$ Tasaki lattice as $\square$ and the square lattice as $\square$. (b) Kagome lattice (left), an example of the line graph construction [80]. A CLS is localized on a hexagon. Every CLS overlaps with $2 f=6$ other CLSs, and the corresponding AKLT model lives on a triangular lattice (right). We symbolize the kagome lattice as $\bowtie$ and the triangular lattice as $\triangle$. An optical kagome lattice has been realized experimentally [89]. (c) 3D Tasaki lattice (left), another example of the cell construction [55,56]. A CLS is localized on seven sites, as covered by the octahedron. Every CLS overlaps with $2 f=6$ other CLSs, the corresponding AKLT model thus lives on a cubic lattice (right). We symbolize the cubic lattice as $\mathbb{E}$.

single-particle ground state degeneracy of $\hat{H}_{\text {hop }}^{f, X}$ is $N(2 f+$ $1)$. The corresponding CLSs are localized on $N$ different positions and are related to each other by lattice translation vectors [90]. The shapes of some CLSs are shown in Fig. 4 and Fig. 5. Let $\left(\hat{B}_{j, \alpha}^{f, X}\right)^{\dagger}$ be the creation operator of a CLS, where $j=1,2, \ldots, N$ labels different positions. A fully packed state (FPS) on $X$ is defined as a product of $N$ CLSs: $\left(\hat{B}_{1, \alpha_{1}}^{f, X}\right)^{\dagger}\left(\hat{B}_{2, \alpha_{2}}^{f, X}\right)^{\dagger} \cdots\left(\hat{B}_{N, \alpha_{N}}^{f, X}\right)^{\dagger} \mid$ vac $\rangle$. In an FPS, the lattice is "fully packed" by $N$ particles. For example, $|\boldsymbol{\beta}\rangle$ in Eq. (12) is an FPS in the sawtooth chain.

We now consider another lattice $X^{\prime}=\left(\Lambda_{X^{\prime}}, \mathscr{B}_{X^{\prime}}\right)$ with $\left|\Lambda_{X^{\prime}}\right|=N$, and each site $\boldsymbol{j} \in \Lambda_{X^{\prime}}$ represents a CLS in the FPS of $X$. Two sites in $X^{\prime}$ are directly connected iff the two corresponding CLSs in the FPS (partially) overlap. For example, as shown in Fig. 5, if $X$ is the 2D (3D) Tasaki lattice, $X^{\prime}$ will be the square (cubic) lattice, while if $X$ is the kagome lattice, $X^{\prime}$ will then be the triangular lattice. In the following, we require that $f$ and $X$ are chosen such that $X^{\prime}$ satisfies the condition $\left|\left\{\boldsymbol{j}^{\prime} \in \Lambda_{X^{\prime}} \mid\left\{\boldsymbol{j}, \boldsymbol{j}^{\prime}\right\} \in \mathscr{B}_{X^{\prime}}\right\}\right|=2 f, \forall \boldsymbol{j} \in \Lambda_{X^{\prime}}$. Define $\Lambda_{X}^{[k]} \subset \Lambda_{X}$ as a set of sites where $k$ CLSs in the FPS overlap. For example, in the sawtooth chain $\Lambda_{X}=\Lambda_{X}^{[1]} \cup \Lambda_{X}^{[2]}$, where $\Lambda_{X}^{[1]}$ is the set of all the bottom sites and $\Lambda_{X}^{[2]}$ is all the top sites. We further require that every site $r \in \Lambda_{X}$ is shared by no more than two CLSs in the FPS, i.e., $\Lambda_{X}^{[k]}=\emptyset$ for $k>2$. We then define the spin model $\hat{H}_{\mathrm{AKLT}}^{f, X^{\prime}}$ on $X^{\prime}$, as introduced in Sec. III A.

The $s$-wave interaction between two spin- $f$ bosons at position $\boldsymbol{r}$ is given by $\sum_{S=0,2, \ldots, 2 f} g_{S, \boldsymbol{r}} \hat{P}_{\boldsymbol{r}}^{(S)}$, where the $\mathrm{SO}(3)$-invariant operator $\hat{P}_{r}^{(S)}$ projects the state onto total spin $S=$ even and satisfies the "completeness relation" $\sum_{S} \hat{P}_{r}^{(S)}=\hat{n}_{\boldsymbol{r}}\left(\hat{n}_{\boldsymbol{r}}-1\right) / 2$ [33]. (For interaction between alkalimetal atoms, it is sufficient to consider the short-range $s$-wave scattering [33].) Spin- $f$ bosons in optical lattices are described by the spin- $f$ Bose-Hubbard model. On the lattice $X$, the model is given by

$$
\begin{aligned}
\hat{H}^{f, X} & :=\hat{H}_{\text {hop }}^{f, X}+\hat{H}_{\text {int }}^{f, X}, \\
\hat{H}_{\text {int }}^{f, X} & :=\sum_{r \in \Lambda_{X}} \sum_{S=0,2, \ldots, 2 f} g_{S, r} \hat{P}_{r}^{(S)} .
\end{aligned}
$$

The $s$-wave scattering Hamiltonian is reminiscent of the AKLT Hamiltonians. If $N$ spin- $f$ bosons are loaded on $X$ and $g_{2 f, \boldsymbol{r}}>0$ and $g_{S<2 f, \boldsymbol{r}}=0$ for all $\boldsymbol{r} \in \Lambda_{X}^{[2]}$, following Sec. II B, the zero-energy ground states of $\hat{H}^{f, X}$ in Eq. (28) and $\hat{H}_{\text {AKLT }}^{f, X^{\prime}}$ in Eq. (25) can thus be exactly mapped to each other, just as in Eq. (15). See Appendix F for discussions of the uniqueness of the ground state of $\hat{H}^{f, X}$.

Let us see some concrete examples. In $d=1$ dimension, besides the sawtooth chain, spin-1 bosons can be loaded on the lattices in Fig. 4 as well. In $d=2$, the 2D Tasaki lattice matches spin- 2 bosons, and the corresponding spin-2 AKLT model lives on a square lattice with the VBS ground state in Fig. 3(b). The kagome lattice is suitable for spin-3 bosons, while the corresponding AKLT model has the spin-3 VBS ground state on a triangular lattice as shown in Fig. 3(c). On the other hand, spin-3 bosons are also compatible with the 3D Tasaki lattice, which corresponds to a 3D spin-3 VBS state in Fig. 3(d). In fact, the Tasaki lattice can be constructed in any dimension, and the sawtooth chain can actually be regarded as the 1D Tasaki lattice $[55,56]$. In general, the $d$-dimensional Tasaki lattice matches spin- $d$ bosons, and the corresponding AKLT model lives on a $d$-dimensional hypercubic lattice. 
(a)

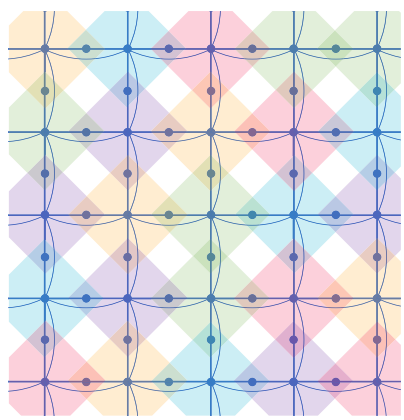

(b)

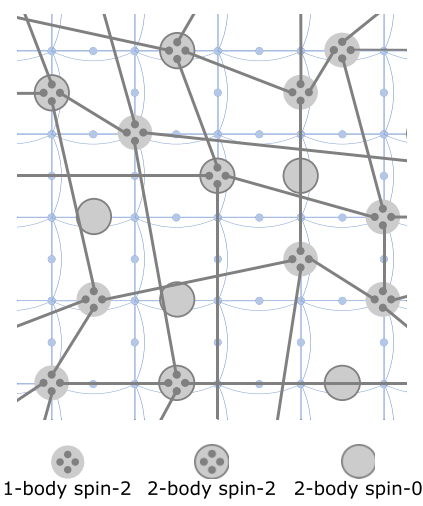

FIG. 6. Components of $\left|\mathrm{GS}_{2, \square}\right\rangle$, the exact ground state of spin-2 bosons on the 2D Tasaki lattice. (a) Visualization of Eq. (30) with a typical choice of $\alpha_{1}, \ldots, \alpha_{N}$. The five possible values of $\alpha_{j}$ are represented by five different colors. Equation (30) is a fully packed state (FPS). (b) Visualization of Eq. (31) with a typical choice of $\boldsymbol{r}_{1}, \ldots, \boldsymbol{r}_{N}$. The state exhibits "hidden VBS order": if we ignore all two-body spin singlets, the remaining particles form a VBS state. The ground state can be viewed as a linear combination of such states with different charge configurations.

Let $\left|\mathrm{GS}_{f, X}\right\rangle$ be the exact and unique ground state of $\hat{H}^{f, X}$. In terms of the Fock basis, $\left|\mathrm{GS}_{f, X}\right\rangle$ reads

$$
\begin{aligned}
\left|\mathrm{GS}_{f, X}\right\rangle= & \sum_{\alpha_{1}, \ldots, \alpha_{N}=-f}^{f} S_{\alpha_{1}, \ldots, \alpha_{N}} \sum_{\boldsymbol{r}_{1}, \ldots, \boldsymbol{r}_{N}} C_{\boldsymbol{r}_{1}, \ldots, \boldsymbol{r}_{N}} \\
& \times \hat{a}_{\boldsymbol{r}_{1}, \alpha_{1}}^{\dagger} \cdots \hat{a}_{\boldsymbol{r}_{N}, \alpha_{N}}^{\dagger}|\mathrm{vac}\rangle,
\end{aligned}
$$

where $S_{\alpha_{1}, \ldots, \alpha_{N}}$ and $C_{\boldsymbol{r}_{1}, \ldots, \boldsymbol{r}_{N}}$ are coefficients that correspond to different spin and charge configurations, respectively. Summing over the charge DOF first gives an FPS:

$$
\begin{aligned}
& \sum_{\boldsymbol{r}_{1}, \ldots, \boldsymbol{r}_{N}} C_{\boldsymbol{r}_{1}, \ldots, \boldsymbol{r}_{N}} \hat{a}_{\boldsymbol{r}_{1}, \alpha_{1}}^{\dagger} \cdots \hat{a}_{\boldsymbol{r}_{N}, \alpha_{N}}^{\dagger}|\mathrm{vac}\rangle \\
& \quad=\left(\hat{B}_{1, \alpha_{1}}^{f, X}\right)^{\dagger}\left(\hat{B}_{2, \alpha_{2}}^{f, X}\right)^{\dagger} \cdots\left(\hat{B}_{N, \alpha_{N}}^{f, X}\right)^{\dagger}|\mathrm{vac}\rangle .
\end{aligned}
$$

$\left|\mathrm{GS}_{f, X}\right\rangle$ is a linear combination of FPSs with different spin configurations, and the coefficients $S_{\alpha_{1}, \ldots, \alpha_{N}}$ in Eq. (29) and Eq. (26) are identical. However, if we sum over the spin DOF first:

$$
\sum_{\alpha_{1}, \ldots, \alpha_{N}=-f}^{f} S_{\alpha_{1}, \ldots, \alpha_{N}} \hat{a}_{\boldsymbol{r}_{1}, \alpha_{1}}^{\dagger} \cdots \hat{a}_{\boldsymbol{r}_{N}, \alpha_{N}}^{\dagger}|\mathrm{vac}\rangle,
$$

we will get a state with "hidden VBS order." $\left|\mathrm{GS}_{f, X}\right\rangle$ can then be alternatively viewed as a linear combination of such states with different charge configurations. The above analysis suggests that the FPS and "hidden VBS order" are two different but equivalent pictures of understanding the structure of the ground states, and they together reflect spin and charge fluctuations at zero temperature. Figure 6 gives examples of an FPS and a state with "hidden VBS order" of spin-2 bosons on the 2D Tasaki lattice.

Let us note that although an exact ground state $\left|\mathrm{GS}_{f, X}\right\rangle$ is a result of fine-tuned interactions, one can readily believe the quantum phase represented by $\left|\mathrm{GS}_{f, X}\right\rangle$ (to be discussed in Sec. IIIC) exists in rather broad parameter regions, as supported by the evidences shown in Sec. IID and IIE for the sawtooth chain.

\section{Classifying the ground states from the viewpoint of SPT phases}

As the unique ground state, $\left|\mathrm{GS}_{f, X}\right\rangle$ preserves all the symmetries of the system. One can always think of $\left|G_{f, X}\right\rangle$ as a representative state of a certain disordered, gapped, short-range entangled, and symmetry-protected quantum phase. In order to classify the phases represented by $\left|\mathrm{GS}_{f, X}\right\rangle$ with various $f$ and $X$, there are two main questions that we need to answer. First, what is the phase of the corresponding VBS state $\left|\mathrm{VBS}_{f, X^{\prime}}\right\rangle$ ? Second, are the two states $\left|\mathrm{GS}_{f, X}\right\rangle$ and $\left|\mathrm{VBS}_{f, X^{\prime}}\right\rangle$ in exactly the same phase?

Recall that for $f=1$ and $X$ being the sawtooth chain, the answers to the two questions have been completely listed in Table I. The two states Eq. (19) and Eq. (8) are in the same phase except when the inversion symmetry is involved. In $d>1$ dimensions, however, regarding the first question, given an arbitrary $f$ and $X^{\prime}$, there is so far no complete answer about the phase of $\left|\mathrm{VBS}_{f, X^{\prime}}\right\rangle$ in terms of all of its symmetry groups. Nevertheless, it has been known that with on-site symmetry [91] alone, $\left|\mathrm{VBS}_{f, X^{\prime}}\right\rangle$ always represents a trivial phase in $d>1$ dimensions, while the combination of certain on-site and spatial symmetry can give an SPT/trivial classification, as will be discussed in Sec. IIIC3. In addition, we will show in Sec. IIIC2 that crystalline symmetries alone can also give an SPT/trivial classification for $\left|\mathrm{VBS}_{f, X^{\prime}}\right\rangle$. Regarding the second question, we claim that $\left|\mathrm{GS}_{f, X}\right\rangle$ and $\left|\mathrm{VBS}_{f, X^{\prime}}\right\rangle$ are always in the same phase protected by on-site symmetry alone or the combination of on-site and translation symmetry; see Sec. IIIC1. However, their phases should be investigated on a case-by-case basis when crystalline symmetries come into play; see Secs. IIIC2 and IIIC3. In particular, we find that the charge fluctuations in $\left|\mathrm{GS}_{f, X}\right\rangle$ can play a nontrivial role in the SPT orders protected by crystalline symmetries. In the following, for simplicity, we focus only on several concrete examples. The analysis, however, applies to general cases.

\section{Smooth path argument}

In terms of the combination of $\mathrm{SO}(3)$ spin rotation and translation symmetry [denote the symmetry group as $\mathrm{SO}(3) \times \operatorname{trn}]$, the spin- $2 \mathrm{VBS}$ state on a square lattice $\left|\mathrm{VBS}_{2, \square}\right\rangle$ is in an SPT phase [92], while the spin-3 VBS state on a triangular lattice $\left|\mathrm{VBS}_{3, \Delta}\right\rangle$ represents a trivial phase [93]. A key observation is that $\left|\mathrm{GS}_{f, X}\right\rangle$ can always be smoothly deformed to $\left|\mathrm{VBS}_{f, X^{\prime}}\right\rangle$ without breaking the $\mathrm{SO}(3) \times$ trn symmetry. Therefore, the two states are in the same phase. For example, let $\hat{B}_{1, \alpha}^{3,}$ be one of the CLS operators on the kagome lattice, whose exact form is given by

$$
\hat{B}_{1, \alpha}^{3, \bigotimes}=\frac{1}{\sqrt{6}} \sum_{x=1}^{6}(-1)^{x} \hat{a}_{x, \alpha},
$$




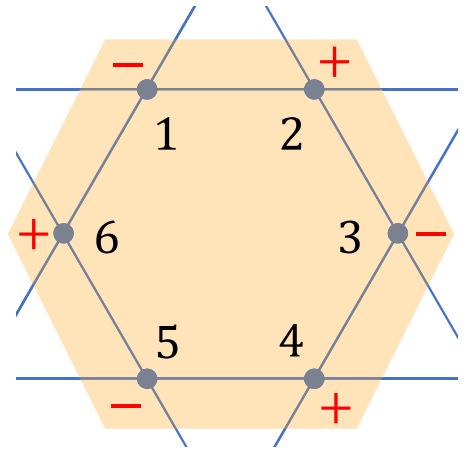

FIG. 7. A CLS in the kagome lattice. Sign of the amplitude alternates from site 1 to 6 .

where the six sites labeled by $x$ form vertices of a hexagon, as shown in Fig. 7. We then define a $\lambda$-deformed CLS operator as

$$
\hat{B}_{1, \alpha}^{3, \nless}(\lambda)=\frac{1}{\sqrt{\lambda^{2}+5}}\left(\sum_{x=1}^{5}(-1)^{x} \hat{a}_{x, \alpha}+\lambda \hat{a}_{6, \alpha}\right),
$$

which satisfies $\hat{B}_{1, \alpha}^{3, \bigotimes}(1)=\hat{B}_{1, \alpha}^{3, \nless}$ and $\lim _{\lambda \rightarrow \infty} \hat{B}_{1, \alpha}^{3, \bigotimes}(\lambda)=\hat{a}_{6, \alpha}$. By applying lattice translation vectors, we can get all the other $\hat{B}_{j, \alpha}^{3, \notin}(\lambda)$ with $j=2, \ldots, N$. Now consider the state defined on the kagome lattice

$$
\left|\mathrm{GS}_{3, \bigotimes}(\lambda)\right\rangle=\sum_{\alpha_{1}, \ldots, \alpha_{N}=-3}^{3} S_{\alpha_{1}, \ldots, \alpha_{N}} \prod_{j=1}^{N}\left(\hat{B}_{j, \alpha_{j}}^{3, \downarrow}(\lambda)\right)^{\dagger}|\mathrm{vac}\rangle,
$$

where $\left\{S_{\alpha_{1}, \ldots, \alpha_{N}}\right\}$ are chosen such that $\left|\mathrm{GS}_{3, \bigotimes}(1)\right\rangle=\left|\mathrm{GS}_{3, \downarrow}\right\rangle$ is the original ground state of $\hat{H}^{3, \star}$. One can then easily see that $\lim _{\lambda \rightarrow \infty}\left|\mathrm{GS}_{3 .}(\infty)\right\rangle=\left|\mathrm{VBS}_{3, \Delta}\right\rangle$ and the state $\left|\mathrm{GS}_{3,}(\lambda)\right\rangle$ remains $\mathrm{SO}(3) \times$ trn symmetric and short-range entangled for $1<\lambda<\infty$. Therefore, $\left|\mathrm{GS}_{3 \nless ぬ}\right\rangle$ and $\left|\mathrm{VBS}_{3, \Delta}\right\rangle$ are smoothly connected and are in the same trivial phase protected by $\mathrm{SO}(3) \times \operatorname{trn}$. For an arbitrary $f$ and $X$, a smooth path between $\left|\mathrm{GS}_{f, X}\right\rangle$ and $\left|\mathrm{VBS}_{f, X^{\prime}}\right\rangle$ can always be explicitly constructed by smoothly deforming every CLS in $X$ to one single site while preserving the $\mathrm{SO}(3)$ or $\mathrm{SO}(3) \times \operatorname{trn}$ symmetry, and thus the two states always represent the same phase protected by the symmetry. Let $\left|\mathrm{GS}_{2, \square}\right\rangle$ be the exact ground state of spin-2 bosons in the 2D Tasaki lattice; for the above reason, $\left|\mathrm{GS}_{2, \square}\right\rangle$ and $\left|\mathrm{VBS}_{2, \square}\right\rangle$ are in the same SPT phase protected by $\mathrm{SO}(3) \times \operatorname{trn}$.

Table III summarizes current results. The purpose of this section (Sec. IIIC1) is to demonstrate the smooth path ar-

TABLE III. In terms of on-site symmetry or on-site $\times$ trn symmetry, $\left|\mathrm{GS}_{f, X}\right\rangle$ and $\left|\mathrm{VBS}_{f, X^{\prime}}\right\rangle$ are always in the same phase.

\begin{tabular}{lcc}
\hline \hline & $\mathrm{SO}(3)$ & $\mathrm{SO}(3) \times \operatorname{trn}$ \\
\hline$\left|\mathrm{VBS}_{2, \square}\right\rangle$ & Trivial & SPT \\
$\left|\mathrm{GS}_{2, \square}\right\rangle$ & & Trivial \\
\hline$\left|\mathrm{VBS}_{3, \Delta}\right\rangle$ & Trivial & \\
$\left|\mathrm{GS}_{3, \downarrow}\right\rangle$ & & \\
\hline \hline
\end{tabular}

(a)

$$
\begin{aligned}
& \underset{a \rightarrow b}{a}=\frac{1}{\sqrt{2}}\left(\left|\uparrow_{a}\right\rangle\left|\downarrow_{b}\right\rangle-\left|\downarrow_{a}\right\rangle\left|\uparrow_{b}\right\rangle\right) \\
& \underset{a}{a} \stackrel{b}{b}=\frac{1}{\sqrt{2}}\left(\left|\downarrow_{a}\right\rangle\left|\uparrow_{b}\right\rangle-\left|\uparrow_{a}\right\rangle\left|\downarrow_{b}\right\rangle\right)
\end{aligned}
$$

(b)

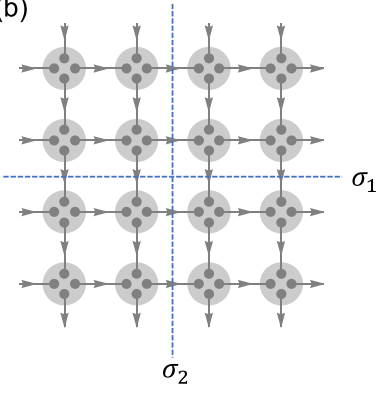

(c)

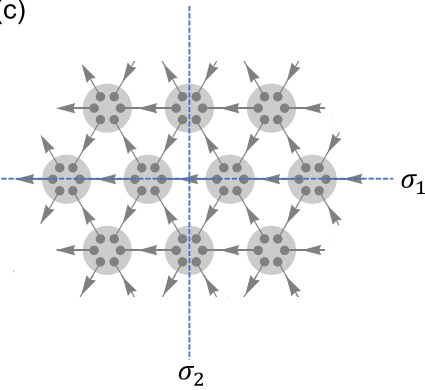

(d)

\begin{tabular}{lcccc}
\hline \hline & id & $\sigma_{1}$ & $\sigma_{2}$ & $\sigma_{1} \sigma_{2}$ \\
\hline$\left|\mathrm{VBS}_{2, \square}\right\rangle$ & 1 & 1 & 1 & 1 \\
$\left|\mathrm{VBS}_{3, \triangle}\right\rangle$ & 1 & 1 & -1 & -1 \\
\hline \hline
\end{tabular}

FIG. 8. (a) A direction can be assigned to each singlet bond. Two opposite directions differ by a minus sign. (b) $\left|\mathrm{VBS}_{2, \square}\right\rangle$ is invariant under reflections about mirror planes $\sigma_{1}$ and $\sigma_{2}$. We assume the symmetry center lies in a plaquette. (c) $\left|\mathrm{VBS}_{3, \Delta}\right\rangle$ is invariant under reflections about mirror planes $\sigma_{1}$ and $\sigma_{2}$. (d) $\left|\mathrm{VBS}_{2, \square}\right\rangle$ results in a trivial representation of $D_{2}=\left\{\mathrm{id}, \sigma_{1}, \sigma_{2}, \sigma_{1} \sigma_{2}\right\}$ (plaquette-centered), while $\left|\mathrm{VBS}_{3, \Delta}\right\rangle$ results in a nontrivial representation of $D_{2}$ (bondcentered).

gument. Details behind the on-site $\times$ trn symmetry will be discussed later (see Sec. IIIC3 and Appendix G).

\section{SPT/trivial phases protected by crystalline symmetries alone}

For general crystalline symmetries, however, the smooth path argument does not always apply. For simplicity, we consider only point group symmetries in $d=2$ dimension in this article. Let $G$ be a point group of a Hamiltonian with a unique gapped ground state $|\Psi\rangle$. Let $\hat{U}(q)$ be the symmetry operation (on the Hilbert space) corresponding to the group element $q \in G$. Subjected to $q$, the unique ground state transforms as

$$
|\Psi\rangle \rightarrow \hat{U}(q)|\Psi\rangle=e^{i \theta_{q}}|\Psi\rangle,
$$

where the phase factors $\left\{e^{i \theta_{q}}\right\}_{q \in G}$ form a 1D representation of $G$, and different 1D representations label different phases protected by the point group $G$ [94]. When $\left\{e^{i \theta_{q}}\right\}_{q \in G}$ is a trivial representation, that is, $e^{i \theta_{q}}=1$ for all $q \in G,|\Psi\rangle$ is in a trivial phase. On the other hand, $|\Psi\rangle$ is in an SPT phase if $\left\{e^{i \theta_{q}}\right\}_{q \in G}$ is a nontrivial representation $[69,95,96]$. It is important to be aware that for point group symmetries alone in $d<3$ dimensions, the SPT/trivial classifications become meaningless when there are microscopic DOF lying precisely at symmetry centers. See Sec. I B of Ref. [95] or Appendix A of Ref. [69] for details. In other words, it is only legal to put the symmetry centers in vacuum.

In the graph representation of VBS states, we can assign an arbitrary direction to each singlet bond, because a singlet state is antisymmetric. Reversing the direction of a singlet bond is equivalent to adding a minus sign; see Fig. 8(a). Let us consider the point group $D_{2}$ as a simple example. Elements of 
$D_{2}$ are generated by two perpendicular mirror planes $\sigma_{1}$ and $\sigma_{2}$, as shown in Figs. 8(b)-8(c). Consider the states $\left|\mathrm{VBS}_{2, \square}\right\rangle$ and $\left|\mathrm{VBS}_{3, \Delta}\right\rangle$ in the thermodynamic limit. $\left|\mathrm{VBS}_{2, \square}\right\rangle$ is $D_{2}-$ symmetric around the center of a plaquette, the center of a bond, or a site. $\left|\mathrm{VBS}_{3, \Delta}\right\rangle$ is $D_{2}$-symmetric around the center of a bond or a site. As emphasized above, to classify their phases, it is illegal to put the symmetry center on a site. For $\left|\mathrm{VBS}_{2, \square}\right\rangle$ with a plaquette-centered $D_{2}$ symmetry, there are an even number [97] of singlet bonds being reversed by a mirror reflection [see Fig. 8(b)], thus $\hat{U}(q)\left|\mathrm{VBS}_{2, \square}\right\rangle=\left|\mathrm{VBS}_{2, \square}\right\rangle$, $\forall q \in D_{2}$. However, for a bond-centered $D_{2}$ symmetry, with respect to the mirror plane perpendicular to the central bond, there are an odd number of singlet bonds being reversed, which results in a nontrivial representation of $D_{2}$. We see that in this example, the phase depends on the position of the symmetry center [98]. For some reason that will be clear later, we consider only the plaquette-centered symmetry for $\left|\mathrm{VBS}_{2, \square}\right\rangle$ in the remnant of this article. For $\left|\mathrm{VBS}_{3, \Delta}\right\rangle$, the bond-centered symmetry is the only legal choice. As shown in Fig. 8(c), the mirror reflection $\sigma_{2}$ reverses an odd number of bonds, we thus have $\hat{U}\left(\sigma_{2}\right)\left|\mathrm{VBS}_{3, \Delta}\right\rangle=-\left|\mathrm{VBS}_{3, \Delta}\right\rangle$. As listed in Fig. 8(d), $\left|\mathrm{VBS}_{2, \square}\right\rangle$ results in a trivial representation of plaquette-centered $D_{2}$ and is therefore in a trivial phase, while $\left|\mathrm{VBS}_{3, \Delta}\right\rangle$ is in an SPT phase protected by $D_{2}$. Note that from the above discussion, one might naively think that one single mirror plane alone (point group $D_{1}=\mathbb{Z}_{2}^{P}$ ) is sufficient to distinguish the SPT from the trivial phase, which is indeed true in $d=1,3$ dimensions [5,69]. However, in the $d=2$ dimension, $D_{1}$ symmetry alone can give only a trivial phase; see Ref. [95].

In general, a smooth path between $\left|\mathrm{GS}_{f, X}\right\rangle$ and $\left|\mathrm{VBS}_{f, X^{\prime}}\right\rangle$ that preserve on-site $x$ trn symmetry may or may not break crystalline symmetries. For example, by smoothly deforming every CLS into the single site at its center, $\left|\mathrm{GS}_{2, \triangleright}\right\rangle$ reduces to $\left|\mathrm{VBS}_{2, \square}\right\rangle$ while preserving the plaquette-centered $D_{2}$ symmetry; see Fig. 6(a). (Though $\left|\mathrm{GS}_{2, \nabla}\right\rangle$ also has site-centered $D_{2}$ symmetry, such symmetry does not give a phase classification.) For $\left|\mathrm{GS}_{3, \downarrow}\right\rangle$, however, the smooth path described by Eq. (34) breaks the $D_{2}$ symmetry. In general, when we are not able to find a path that is both crystalline-symmetrypreserving and smooth, such a path either is too complicated to be explicitly found or simply does not exist. Nevertheless, it is always possible to investigate the crystalline-symmetryprotected phase of $\left|\mathrm{GS}_{f, X}\right\rangle$ case-by-case. We again use $\left|\mathrm{GS}_{3 .}\right\rangle$ as an example. As shown in Fig. 9(a), we put the symmetry center at the geometric center of a hexagonal plaquette. A CLS in the kagome lattice can actually be regarded as a zero-dimensional SPT phase protected by $D_{2}$, because, for example, according to Eq. (32) and Fig. 7, $\hat{U}\left(\sigma_{2}\right) \hat{B}_{1, \alpha}^{3, \nless} \hat{U}\left(\sigma_{2}\right)=$ $-\hat{B}_{1, \alpha}^{3,}$. The many-body ground state $\left|\mathrm{GS}_{3, \downarrow}\right\rangle$ is a fully packing of CLSs with entangled spin DOF. The spin configurations of $\left|\mathrm{GS}_{3, \downarrow}\right\rangle$, which is inherited from $\left|\mathrm{VBS}_{3, \Delta}\right\rangle$, transforms trivially, as shown in Figs. 9(b) and 9(c). [Note that Fig. 9(b) does not imply that $\left|\mathrm{VBS}_{3, \Delta}\right\rangle$ is in a trivial phase protected by $D_{2}$, as the symmetry is site-centered.] Nevertheless, $\left|\mathrm{GS}_{3 \notin 亠}\right\rangle$ yields a nontrivial representation of $D_{2}$ thanks to how CLSs transform. We thus see that $\left|\mathrm{GS}_{3, \bigotimes}\right\rangle$ is in an SPT phase protected by $D_{2}$; the SPT phase is purely a result of charge fluctuations at zero temperature, as the spin DOF contribute
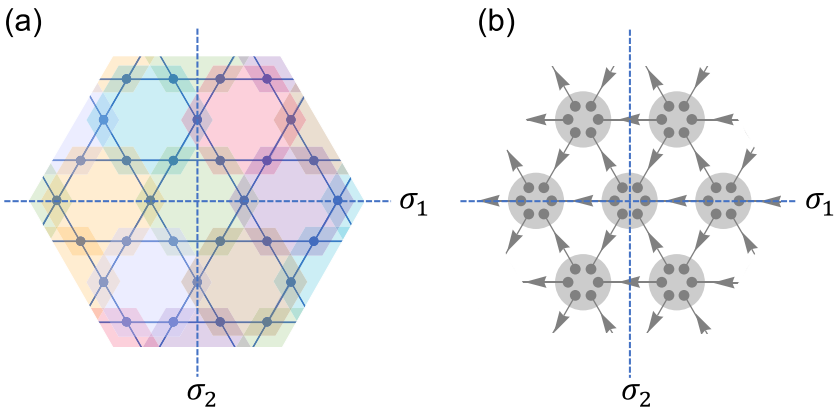

(c)

\begin{tabular}{lcccc}
\hline \hline & id & $\sigma_{1}$ & $\sigma_{2}$ & $\sigma_{1} \sigma_{2}$ \\
\hline$\left|\mathrm{GS}_{3, \not \downarrow}\right\rangle$ & 1 & 1 & -1 & -1 \\
$\left|\mathrm{VBS}_{3, \triangle}\right\rangle$ & 1 & 1 & 1 & 1 \\
\hline \hline
\end{tabular}

FIG. 9. (a) An FPS on the kagome lattice. $\left(\left|\mathrm{GS}_{3}\right\rangle\right.$ is $D_{2}$ symmetric, and it is a superposition of FPSs with different spin configurations.) We require that the symmetry center of $D_{2}$ lies at the geometric center of a hexagon. (b) For $\left|\mathrm{VBS}_{3, \Delta}\right\rangle$, the symmetry center should lie at a site (spin) in order to be compatible with (a). (c) 1D representations of $D_{2}$ associated with $\left|\mathrm{GS}_{3} \star\right\rangle$ and $\left|\mathrm{VBS}_{3, \Delta}\right\rangle$. Note that the latter state transforms trivially, but this does not mean that it is in a trivial phase.

trivially. Similarly, with the symmetry center in Fig. 9(a), one can also show that $\left.\left|\mathrm{GS}_{3}\right\rangle\right\rangle$ represents an SPT phase protected by point group $D_{3}$ or $D_{6}$, while $D_{3}$ and $D_{6}$ are not a proper symmetries for the phase of $\left|\mathrm{VBS}_{3, \Delta}\right\rangle$, since the VBS state is $D_{3}$ or $D_{6}$ invariant only about a site. Once again, the SPT phase of $\left|\mathrm{GS}_{3, \downarrow}\right\rangle$ protected by $D_{3}$ or $D_{6}$ originates from the charge fluctuations of each CLS. Results of the current section are summarized in Table IV.

For a specific symmetry, to identify the phase of $\left|\mathrm{GS}_{f, X}\right\rangle$, we can first try to find a both symmetry-preserving and smooth path that connects $\left|\mathrm{GS}_{f, X}\right\rangle$ to $\left|\mathrm{VBS}_{f, X^{\prime}}\right\rangle$, provided that the phase of $\left|\mathrm{VBS}_{f, X^{\prime}}\right\rangle$ is already known. (For point group symmetries, we require that there are no microscopic DOF lying at the symmetry center all along the path [99].) When such a path cannot be explicitly found, it is either too complicated to be found or simply absent. Nevertheless, for point group symmetries, based on Eq. (35), one can always classify the phase of $\left|\mathrm{GS}_{f, X}\right\rangle$ without the help of the smooth path argument.

TABLE IV. Classifying the phases of $2 \mathrm{D}$ bosonic states in terms of point group symmetries. Symmetry centers of the points groups are placed in vacuum. In addition, $D_{2}$ and $D_{4}$ are assumed to be plaquette-centered symmetries for $\left|\mathrm{VBS}_{2, \square}\right\rangle . \mathrm{N} / \mathrm{A}$ means either that the state does not have such symmetry or that the symmetry can only be site-centered.

\begin{tabular}{lcccc}
\hline \hline & $\left|\mathrm{VBS}_{2, \square}\right\rangle$ & $\left|\mathrm{GS}_{2, \Xi}\right\rangle$ & $\left|\mathrm{VBS}_{3, \Delta}\right\rangle$ & $\left|\mathrm{GS}_{3, \square}\right\rangle$ \\
\hline$D_{1}$ & Trivial & Trivial & Trivial & Trivial \\
$D_{2}$ & Trivial & Trivial & SPT & SPT \\
$D_{3}$ & N/A & N/A & N/A & SPT \\
$D_{4}$ & Trivial & Trivial & N/A & N/A \\
\hline \hline
\end{tabular}


TABLE V. Classifying the phases of $2 \mathrm{D}$ and 3D bosonic states in terms of some on-site $\times$ crystalline symmetries. $D_{1}=\mathbb{Z}_{2}^{P}$ refers to mirror reflection symmetry along an array of sites. All the SPT phases are a result of LSM theorems. The spin-3 VBS state on a cubic lattice $\left|V S_{3, \mathbb{E}}\right\rangle$ represents a trivial phase in terms of $\mathbb{Z}_{2} \times \mathbb{Z}_{2} \times D_{1}$ or TR $\times D_{1}$, because the surface state can be trivially gapped out without breaking these two symmetries. $\left|G_{3,3 \mathrm{DTas}}\right\rangle$ refers to the exact ground state of spin-3 bosons on the 3D Tasaki lattice, and it is smoothly connected to $\left|\mathrm{VBS}_{3, \mathbb{E}}\right\rangle$ while preserving the four symmetries.

\begin{tabular}{lcccc}
\hline \hline & $\left|\mathrm{VBS}_{2, \square}\right\rangle$ & $\left|\mathrm{GS}_{2, \square}\right\rangle$ & $\left|\mathrm{VBS}_{3, \mathbb{E}}\right\rangle$ & $\left|\mathrm{GS}_{3,3 \mathrm{DTa}}\right\rangle$ \\
\hline $\mathbb{Z}_{2} \times \mathbb{Z}_{2} \times$ trn & SPT & SPT & SPT & SPT \\
$\mathrm{TR} \times \operatorname{trn}$ & SPT & SPT & SPT & SPT \\
$\mathbb{Z}_{2} \times \mathbb{Z}_{2} \times D_{1}$ & SPT & SPT & Trivial & Trivial \\
$\mathrm{TR} \times D_{1}$ & SPT & SPT & Trivial & Trivial \\
\hline \hline
\end{tabular}

We would like to mention some related research. The "fragile Mott insulator" studied in Ref. [100] can be understood as SPT phases protected by point group symmetries. In contrast, the "featureless Mott insulator" of spinless bosons studied in Refs. [101,102] should be classified into trivial phases protected by point group symmetries.

\section{SPT phases protected by on-site $x$ crystalline symmetries: A result of the Lieb-Schultz-Mattis (LSM) theorems}

There can also be nontrivial interplay between on-site and crystalline symmetries. For instance, the $\mathrm{SO}(3) \times \operatorname{trn}$ symmetry introduced in Sec. IIIC1 is one such example. In fact, $\mathrm{SO}(3) \times$ trn is sufficient but not necessary to protect the SPT phase of $\left|\mathrm{VBS}_{2, \square}\right\rangle$ : the subgroup $\mathbb{Z}_{2} \times \mathbb{Z}_{2} \times$ trn is enough. Besides, $\left|\mathrm{VBS}_{2, \square}\right\rangle$ is in an SPT phase protected by the combination of time-reversal (TR) and site-centered mirror reflection (= point group $D_{1}=\mathbb{Z}_{2}^{P}$ ) symmetry. Other such symmetries include $\mathrm{TR} \times \operatorname{trn}, \mathbb{Z}_{2} \times \mathbb{Z}_{2} \times D_{1}$, and so on; see Table V. In fact, the reason why certain on-site $\times$ spatial symmetries can give SPT/trivial classifications in $d>$ 1 dimensions is closely related to the LSM theorems; see Appendix $\mathrm{G}$ for details.

As explained in Sec. IIIC1, $\left|\mathrm{GS}_{f, X}\right\rangle$ is always smoothly connected to $\left|\mathrm{VBS}_{f, X^{\prime}}\right\rangle$ while preserving on-site $\times \operatorname{trn}$ symmetries. However, for on-site $x$ point group or on-site $x$ space group symmetries, a symmetry-preserving smooth path may not exist. (Luckily, for $\mathbb{Z}_{2} \times \mathbb{Z}_{2} \times D_{1}$ and $\mathrm{TR} \times D_{1}$, $\left|\mathrm{GS}_{2, \square}\right\rangle$ and $\left|\mathrm{GS}_{3,3 \mathrm{DTas}}\right\rangle$ are smoothly connected to their corresponding VBS states.) In the case where a symmetrypreserving smooth path cannot be found, the phase of $\left|\mathrm{GS}_{f, X}\right\rangle$ can always be judged by examining that if one can trivially gap out the edge state without out breaking the symmetry: $\left|\mathrm{GS}_{f, X}\right\rangle$ is in an SPT phase if its edge state cannot be trivially gapped out.

\section{DISCUSSION}

We show that the SPT phases can be realized with short-range interacting spinful bosons that are loaded on the lattices with a bottom flat band. Such systems are described by the spinful Bose-Hubbard models. The ground states of such systems have both spin and charge fluctuations. The single-body eigenstates of a flat band can usually be chosen to be strictly localized on finite number of sites, known as compact localized states (CLSs). When $N$ spin- $f$ bosons are loaded on a bottom-flat-band lattice $X$ with $N$ unit cells, at low temperatures, the particles' wave functions tend to avoid overlapping each other in order to minimize the system's energy. In particular, when the interaction strength between spin- $f$ bosons is fine-tuned, in the ground state $\left|\mathrm{GS}_{f, X}\right\rangle, N$ bosons exactly occupy $N$ CLSs on different patches. We make use of the analogy between the Hamiltonian that describes the $s$-wave collision among spin- $f$ bosons and the spin- $f$ AKLT Hamiltonian. This analogy enables us to exactly map $\left|\mathrm{GS}_{f, X}\right\rangle$ onto $\left|\mathrm{VBS}_{f, X^{\prime}}\right\rangle$, where the latter state is the spin- $f$ VBS state on the lattice $X^{\prime}$. This implies that $\left|\mathrm{GS}_{f, X}\right\rangle$ is the exact and unique many-body ground state of the spin- $f$ Bose-Hubbard model. The choice of $f$ and $X^{\prime}$ is determined by the geometry of $X$. Note that bottom-flat-band lattices $X$ can be constructed systematically; see Sec. III B.

Over the years, exact results have proved to be highly valuable in quantum and statistical physics. Our work features the exact many-body ground states $\left|\mathrm{GS}_{f, X}\right\rangle$ of spinful itinerant systems. The spin fluctuations of $\left|\mathrm{GS}_{f, X}\right\rangle$ is inherited from $\left|\mathrm{VBS}_{f, X^{\prime}}\right\rangle$. Therefore, with respect to the spin rotation symmetry or the spin rotation $\times$ translation symmetry, the symmetry-protected phase of $\left|\mathrm{GS}_{f, X}\right\rangle$ is identical to that of $\left|\mathrm{VBS}_{f, X^{\prime}}\right\rangle$. However, unlike $\left|\mathrm{VBS}_{f, X^{\prime}}\right\rangle$, the state $\left|\mathrm{GS}_{f, X}\right\rangle$ also possesses nonvanishing charge fluctuations, and in terms of crystalline symmetries, both spin and charge fluctuations in $\left|\mathrm{GS}_{f, X}\right\rangle$ together determine its symmetry-protected phase. Hence, as explained in Sec. IIIC2, one cannot simply conclude that the crystalline-symmetry-protected phase of $\left|\mathrm{GS}_{f, X}\right\rangle$ is also inherited from $\left|\mathrm{VBS}_{f, X^{\prime}}\right\rangle$, because charge fluctuations may play a nontrivial role in the former state, which is indeed the case for spin-3 bosons in the kagome lattice. Although our analysis in $d>1$ dimensions is based on the exact ground states $\left|\mathrm{GS}_{f, X}\right\rangle$ (as a consequence of fine-tuned parameters), we expect that just like what has been shown in the spin-1 BHMSC, the SPT phases survive in wider parameter regions, and $\left|G_{f, X}\right\rangle$ just serves as a representative state of the phases.

We having been ignoring the long-range dipole-dipole interaction (DDI), and this can be justified in many alkali-metal atom experiments. In fact, several kinds of transition-metal atoms can also be regarded as spinful bosons, such as ${ }^{52} \mathrm{Cr}$ (spin-3), ${ }^{164}$ Dy (spin-8), and ${ }^{168} \operatorname{Er}$ (spin-6) [33]. Interestingly, these transition-metal atoms have very strong magnetic DDI [103-105]. It is also known that even for bosonic alkalimetal atoms, the magnetic DDI can have a significant effect in certain cases [33]. When taking the DDI into account (in addition to the short-range $s$-wave collision), it is probably impossible to exactly write down the many-body ground states. Nevertheless, we expect that the DDI induces new phases, such as charge density wave and supersolid, due to its long-range nature. Hunting new phases, including the SPT phases, in itinerant spinful bosonic systems with DDI will be an interesting future direction. Note that systems with magnetic DDI no longer have spin rotation symmetry. Instead, the magnetic DDI is invariant under simultaneous rotation in both spin and real spaces $\hat{V}^{\delta}(\theta):=\exp \left[-i \theta \sum_{r}\left(\hat{S}_{r}^{\delta}+\hat{L}_{r}^{\delta}\right)\right]$, where $\hat{L}_{r}^{\delta}$ is the orbital angular momentum operator in the $\delta(=x, y, z)$-direction for particles at position $\boldsymbol{r}[33,106]$. In 
other words, such systems conserve total angular momentum in free space. When constrained on a lattice, the systems can still preserve some discrete rotation symmetries, though such rotation symmetries are not on-site. In the future studies, it is worth investigating how such symmetries classify the SPT phases.

Finally, we would like to make some remarks about the flat band. The flat band has been gaining much attention these years because it was found to give rise to various collective phenomena, such as ferromagnetism [56,61] and superconductivity [86,107], in quantum many-body systems. In this paper, we discover that the flat band can also be an origin of interacting SPT phases. We believe this work stimulates future research on the relation between flat bands and topological quantum physics.

Interestingly, there exists many kinds of lattices where the flat band appears in the middle or top of the band structure [48,82-84]. One can certainly follow the scheme in this paper to construct the many-body eigenstates in these lattices with the help of the CLSs of the flat bands. The resulting manybody eigenstates, due to their short-range entangled nature, are actually quantum many-body scars [108-112], which lead to weak ergodicity breaking of the systems. Exploring quantum many-body scars in spinful atoms with a flat band will be another intriguing future direction.

\section{ACKNOWLEDGMENTS}

We acknowledge stimulating discussions with Hal Tasaki, Synge Todo, and Linhao Li. H.Y. was supported by Grant-inAid for JSPS Research Fellowship for Young Scientists (DC1) No. 20J20715. H.N. was supported by the Advanced Leading Graduate Course for Photon Science (ALPS) at the University of Tokyo. H.K. was supported in part by JSPS Grant-in-Aid for Scientific Research on Innovative Areas No. JP20H04630, JSPS KAKENHI Grant No. JP18K03445, and the Inamori Foundation.

\section{APPENDIX A: THE HALDANE INSULATOR PHASE}

Spinless bosons in optical lattices with dipole-dipole interaction are described by the extended Bose-Hubbard model. The nature of the Haldane insulator phase in the $1 \mathrm{D}$ extended Bose-Hubbard model can be captured by the following state [13]:

$$
\left|\Psi_{\mathrm{HI}}\right\rangle=\prod_{j}\left(\hat{a}_{j}^{\dagger}+\hat{a}_{j+1}^{\dagger}\right)|\mathrm{vac}\rangle
$$

where $\hat{a}_{j}^{\dagger}$ creates a spinless boson at site $j$; see Fig. 10(a). The state $\left|\Psi_{\mathrm{HI}}\right\rangle$ represents a trivial phase in the sense of inversion symmetry, but it represents a Haldane phase protected by the combination of pseudospin rotation and the inversion symmetry, i.e., the group $\{1, \hat{U}(n \mathcal{I})\}$ with $\hat{U}(n \mathcal{I}):=\exp \left[-i \pi \sum_{r}\left(\hat{n}_{r}-1\right)\right] \hat{U}(\mathcal{I})$. This is the same for $\left|\mathrm{GS}_{\lambda=0}\right\rangle$; see Table I. In addition, the Haldane phase of $\left|\mathrm{GS}_{\lambda=0}\right\rangle$ is protected by other symmetries related to the spin DOF. In this sense, we can say the state $\left|G_{\lambda=0}\right\rangle$ represents a spinful Haldane insulator phase. Note that both $\left|\Psi_{\mathrm{HI}}\right\rangle$ and $\left|\mathrm{GS}_{\lambda=0}\right\rangle$ exhibit perfect hidden charge order, i.e., vacant sites (a)

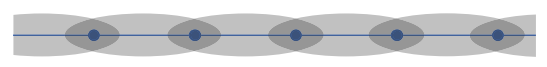

(b)

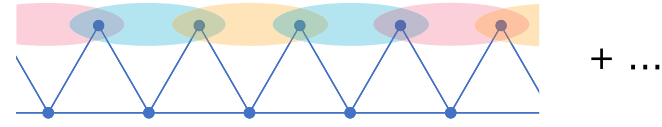

FIG. 10. (a) The Haldane insulator phase in the 1D system of spinless bosons. Every ellipse represents a single particle. (b) The state $|\mathrm{GS}\rangle$ at $\lambda=0$. Three different colors denote three different spin states, and all allowed spin configurations are summed up (with coefficients).

and doubly occupied sites appear alternatively if we ignore all the singly occupied sites.

\section{APPENDIX B: SPIN AND CHARGE EDGE STATES}

For spin-1 BHMSC with OBC, $g_{0}^{\mathrm{t}}=0$ and $g_{2}^{\mathrm{t}}>0$, there are four degenerate ground states $\left|\mathrm{G} \mathrm{S}_{\uparrow \uparrow}\right\rangle,\left|\mathrm{G} S_{\uparrow \downarrow}\right\rangle,\left|\mathrm{GS}_{\downarrow \uparrow}\right\rangle$, and $\left|G S_{\downarrow \downarrow}\right\rangle$, which correspond to four independent edge spin-1/2 states. The analytical forms of $\left\langle\mathrm{GS}_{\uparrow \uparrow}\left|\hat{S}_{r}^{\delta}\right| \mathrm{GS}_{\uparrow \uparrow}\right\rangle$, $\left\langle\mathrm{GS}_{\uparrow \uparrow}\left|\hat{n}_{r}\right| \mathrm{GS} \mathrm{S}_{\uparrow \uparrow}\right\rangle$, etc. are rather complicated and will not be presented. Instead, their plots are shown in Fig. 11. The spin
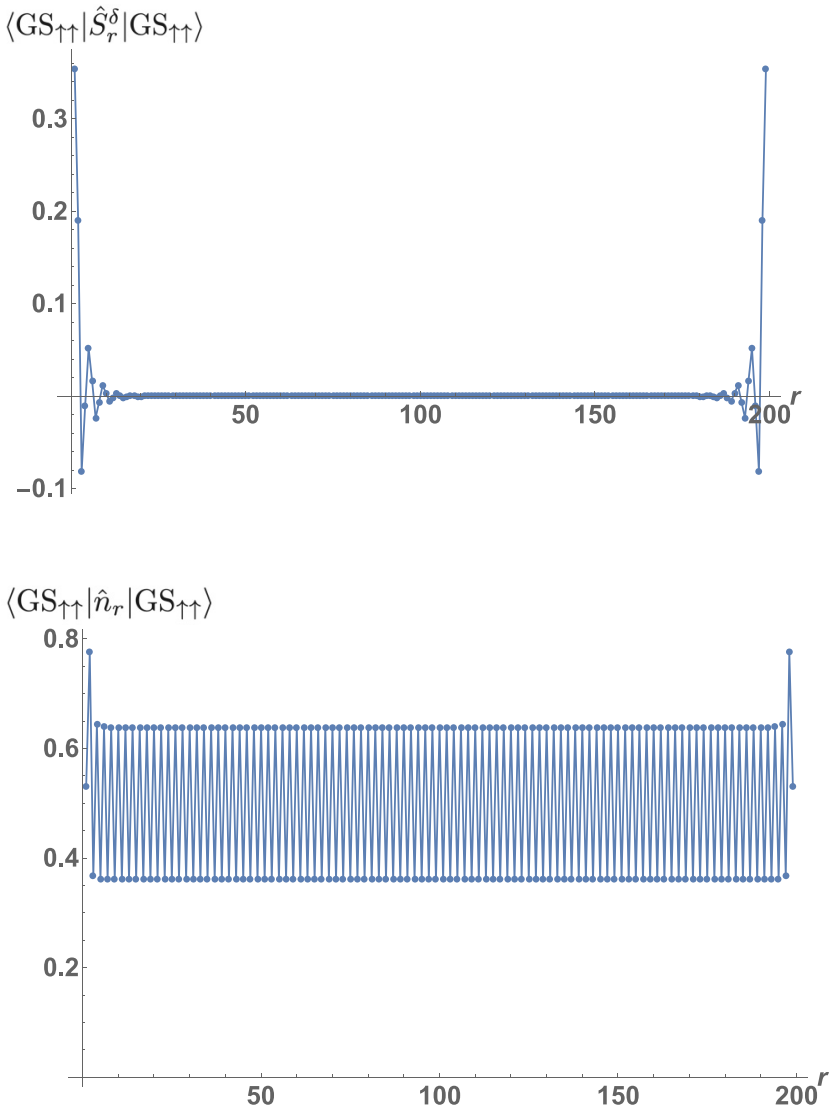

FIG. 11. Spin and charge edge states. We have assumed that the sawtooth chain ends with bottom sites at both ends and taken $N=$ 100 and $\lambda=1$. 


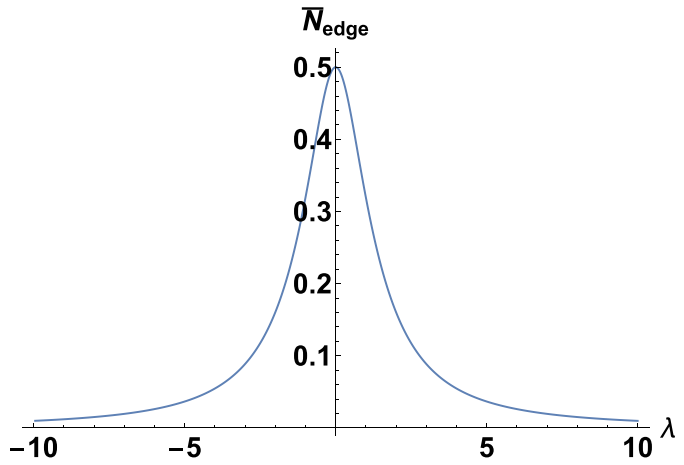

FIG. 12. The reduced edge particle number $\bar{N}_{\text {edge }}$ as a function of $\lambda$.

edge state decays more slowly than the charge one, because the spin correlation length is longer than the charge correlation length:

$$
\begin{aligned}
\xi_{\text {spin }} & =\left(\ln \frac{3 \lambda^{2}+\sqrt{9 \lambda^{4}+36 \lambda^{2}+24}+6}{\lambda^{2}+\sqrt{\lambda^{4}+4 \lambda^{2}+24}+2}\right)^{-1}, \\
\xi_{\text {charge }} & =\left(\ln \frac{3 \lambda^{2}+\sqrt{9 \lambda^{4}+36 \lambda^{2}+24}+6}{3 \lambda^{2}-\sqrt{9 \lambda^{4}+36 \lambda^{2}+24}+6}\right)^{-1} .
\end{aligned}
$$

There is always a spin-1/2 localized at each edge. We define the reduced edge particle number as $\bar{N}_{\text {edge }}:=$ $\sum_{r=1}^{\infty}\left(\lim _{N \rightarrow \infty}\left\langle\mathrm{GS}_{\uparrow \uparrow}\left|\hat{n}_{r}\right| \mathrm{GS}_{\uparrow \uparrow}\right\rangle-1 / 2\right) . \bar{N}_{\text {edge }}$ is a function of $\lambda$, and $\bar{N}_{\text {edge }}=1 / 2$ at $\lambda=0$. See Fig. 12 . Note that $\bar{N}_{\text {edge }}$ is independent of the four degenerate ground states.

Let us note that for crystalline-symmetry-protected topological phases in $d<3$ dimensions, there are no anomalous edge states [4,95]. For example, both $\left|\mathrm{GS}_{3, \bigotimes}\right\rangle$ and $\left|\mathrm{VBS}_{3, \Delta}\right\rangle$ are protected by $D_{2}$ symmetry (see Sec. III), and they do not have anomalous edge states.

\section{APPENDIX C: KENNEDY-TASAKI TRANSFORMATION FOR INTEGER-SPIN ITINERANT SYSTEMS}

The Kennedy-Tasaki transformation is a nonlocal unitary transformation defined on an open chain of length $L$ as $[5,7-9]$

$$
\hat{U}_{\mathrm{KT}}:=\prod_{j=1}^{L} \exp \left[i \pi\left(\sum_{i=1}^{j-1} \hat{S}_{i}^{z}\right) \hat{S}_{j}^{x}\right],
$$

and it is Hermitian: $\hat{U}_{\mathrm{KT}}^{\dagger}=\hat{U}_{\mathrm{KT}}$. $\hat{U}_{\mathrm{KT}}$ is also invariant under $\mathbb{Z}_{2} \times \mathbb{Z}_{2}$ spin rotation. For an arbitrary integer-spin chain, let $\hat{h}_{j}$ be a local Hamiltonian, and the sufficient and necessary condition for $\hat{U}_{\mathrm{KT}} \hat{h}_{j} \hat{U}_{\mathrm{KT}}$ also to be local is that $\hat{h}_{j}$ is $\mathbb{Z}_{2} \times \mathbb{Z}_{2}$ invariant [4,9]. This statement can be extended to integerspin itinerant systems. For example, the spin-1 Bose-Hubbard model has $\mathrm{SO}(3)$ spin rotation symmetry, and the on-site interaction $\hat{P}_{r}^{(S)}$ is invariant under $\hat{U}_{\mathrm{KT}}$, while the $\mathrm{SO}(3)$-invariant hopping transforms as

$$
\begin{gathered}
\hat{U}_{\mathrm{KT}}\left(\sum_{\alpha=0, \pm 1} \hat{a}_{i, \alpha}^{\dagger} \hat{a}_{j, \alpha}+\text { H.c. }\right) \hat{U}_{\mathrm{KT}} \\
=e^{i \pi \sum_{v=i}^{j-1} \hat{S}_{v}^{z} a_{i, 0}^{\dagger} \hat{a}_{j, 0}+\text { H.c. }}
\end{gathered}
$$

TABLE VI. Four different 1D representations of $\mathbb{Z}_{2} \times \mathbb{Z}_{2}$.

\begin{tabular}{lllll}
\hline \hline & $\phi_{1}$ & $\phi_{x}$ & $\phi_{y}$ & $\phi_{z}$ \\
\hline$\gamma=1$ & +1 & +1 & +1 & +1 \\
$\gamma=x$ & +1 & +1 & -1 & -1 \\
$\gamma=y$ & +1 & -1 & +1 & -1 \\
$\gamma=z$ & +1 & -1 & -1 & +1 \\
\hline \hline
\end{tabular}

$$
\begin{aligned}
& +\frac{1}{2} e^{i \pi \sum_{v=i+1}^{j} \hat{S}_{v}^{x}}\left[\left(e^{i \pi \sum_{u=i}^{j-1} \hat{S}_{u}^{z}}+1\right)\left(a_{i,+}^{\dagger} \hat{a}_{j,+}+a_{i,-}^{\dagger} \hat{a}_{j,-}\right)\right. \\
& \left.+\left(e^{i \pi \sum_{u=i}^{j-1} \hat{S}_{u}^{z}}-1\right)\left(\hat{a}_{i,+}^{\dagger} a_{j,-}+\hat{a}_{i,-}^{\dagger} \hat{a}_{j,+}\right)\right]+ \text { H.c. }
\end{aligned}
$$

We can see that the transformed hopping has $\mathbb{Z}_{2} \times \mathbb{Z}_{2}$ symmetry and is still local if the original hopping is local.

\section{APPENDIX D: TRANSLATION SYMMETRY AND THE HALDANE PHASES}

In the presence of both translation symmetry and $\mathbb{Z}_{2} \times \mathbb{Z}_{2}$ symmetry, $\left\{\phi_{q}\right\}_{q \in \mathbb{Z}_{2} \times \mathbb{Z}_{2}}$ in Eq. (22) forms a $1 \mathrm{D}$ representation of the group $\mathbb{Z}_{2} \times \mathbb{Z}_{2}$. In this case, all the phases of gapped states that do not break the two symmetries are classified

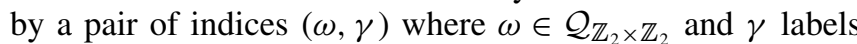
different $1 \mathrm{D}$ representations of $\mathbb{Z}_{2} \times \mathbb{Z}_{2}$ [71]; see Table VI. Let us again use the sawtooth chain as an example. It is easy to see that the state $|\mathrm{GS}\rangle$ in Eq. (19) corresponds to the row $\gamma=1$ in Table VI. We now show that other three SPT phases labeled by $(\omega=-1, \gamma=x, y, z)$ can be obtained by slightly modifying $\hat{H}_{\text {hop }}$. Define

$$
\hat{W}^{\gamma}:=\prod_{k=1}^{N / 4} e^{-i \pi\left(\hat{S}_{4 k-1}^{\gamma}+\hat{S}_{4 k}^{\gamma}\right)}, \quad \gamma=x, y, z .
$$

The operator $\hat{W}^{\gamma}$ acts on the red sites pictured in Fig. 13. Interaction $\hat{H}_{\text {int }}$ is invariant under $\hat{W}^{\gamma}$, thus $\hat{H}^{\gamma}:=\hat{W}^{\gamma} \hat{H} \hat{W}^{\gamma}=$ $\hat{W}^{\gamma} \hat{H}_{\text {hop }} \hat{W}^{\gamma}+\hat{H}_{\text {int }}$. The transformation does not break the translation symmetry. The unique ground state of $\hat{H}^{\gamma}$ is given by

$$
\begin{aligned}
\left|\mathrm{GS}^{\gamma}\right\rangle= & \hat{W}^{\gamma}|\mathrm{GS}\rangle \\
= & \sum_{\tau_{1}, \ldots, \tau_{2 N}=-1}^{3} \operatorname{Tr}\left(F^{\tau_{1}} E^{\tau_{2}} \Sigma^{\gamma} F^{\tau_{3}} E^{\tau_{4}} \Sigma^{\gamma} \ldots\right) \\
& \times\left(\prod_{r=1}^{2 N} \hat{d}_{r, \tau_{r}}^{\dagger}\right)|\mathrm{vac}\rangle,
\end{aligned}
$$

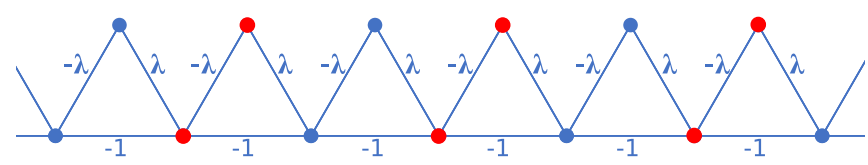

FIG. 13. Hopping constant $t_{r, r^{\prime}}$ for magnetic sublevel \pm 1 in $\hat{W}^{\gamma} \hat{H}_{\text {hop }} \hat{W}^{\gamma}$ when $\gamma=z$. Operator $\hat{W}^{\gamma}$ acts on the red sites. One can see that the transformation $\hat{W}^{\gamma}$ preserves the translation symmetry. 
where $\Sigma^{\gamma}:=\operatorname{diag}\left(\sigma^{\gamma}, \sigma^{\gamma}\right)$. Under the $\mathbb{Z}_{2} \times \mathbb{Z}_{2}$ spin rotation, matrices in $\left|\mathrm{GS}^{\gamma}\right\rangle$ transforms as

$$
F^{\tau_{2 j-1}} E^{\tau_{2 j}} \Sigma^{\gamma} \rightarrow e^{i \phi_{q}} u_{q}^{\dagger} F^{\tau_{2 j-1}} E^{\tau_{2 j}} \Sigma^{\gamma} u_{q} .
$$

Unitary matrices $\left\{u_{q}\right\}$ above are also given in Table I. Explicit calculation yields the other three different 1D representations of $\mathbb{Z}_{2} \times \mathbb{Z}_{2}$ in Table VI. We thus have the desired SPT phases.

\section{APPENDIX E: MPS ANSATZ FOR NUMERICAL CALCULATIONS}

The MPS ansatz used in our numerical calculation can be regarded as a generalization of Eq. (19). For the matrices in Eq. (19), we can assign a pair of quantum numbers to each block as

$$
\begin{aligned}
& \mathcal{F}:=\sqrt{\lambda^{2}+2} \sum_{\tau=-1}^{3} F^{\tau} \hat{d}_{r, \tau}^{\dagger} \\
&=1 / 2\left(\begin{array}{c|c|c}
0 & -\lambda \sum_{\alpha} M^{\alpha} \hat{a}_{r, \alpha}^{\dagger}
\end{array}\right), \\
& \hline 0 \multicolumn{2}{|c|}{I_{2}} 3 / 2 \\
& \mathcal{E}:=\sum_{\tau=-1}^{3} E^{\tau} \hat{d}_{r, \tau}^{\dagger}={ }^{0}\left(\begin{array}{c|c}
\sum_{\alpha} M^{\alpha} \hat{a}_{r, \alpha}^{\dagger} & \sqrt{6} \hat{b}_{r}^{\dagger} I_{2} \\
\hline I_{2} & \sum_{\alpha} M^{\alpha} \hat{a}_{r, \alpha}^{\dagger}
\end{array}\right) .
\end{aligned}
$$

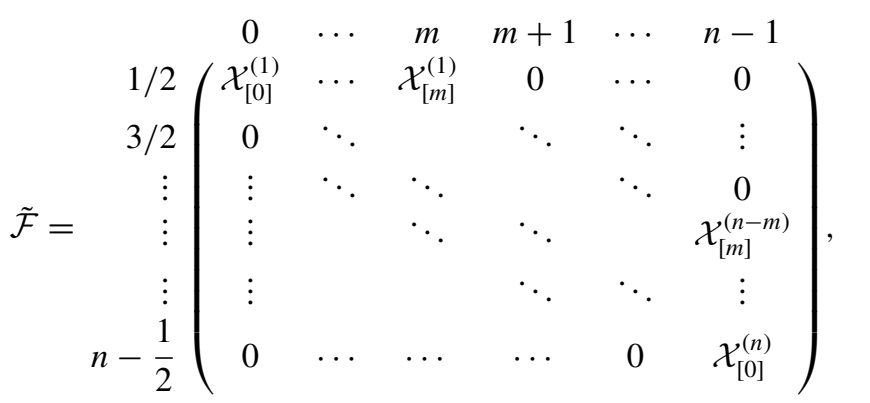

where $\mathcal{X}_{[k]}$ and $\mathcal{Y}_{[k]}$ denote $d \times d$ blocks that create $k$ particles, and the maximum particle number on each site is truncated to $m$. The bond dimension is $\chi=d n$. The $\operatorname{MPS}|\Psi(\tilde{\mathcal{E}}, \tilde{\mathcal{F}})\rangle:=$ $\operatorname{Tr}(\cdots \tilde{\mathcal{E}} \tilde{\mathcal{F}} \tilde{\mathcal{E}} \tilde{\mathcal{F}} \ldots) \mid$ vac $\rangle$ is called a symmetric uniform MPS (suMPS) [72]. In our numerical calculations, we fix $m=3$, $n=4$ and vary $d$. We optimize the suMPS by minimizing its energy expectation value, and the optimization is done by the VUMPS algorithm.

Now we justify the particle number truncation $m=3$. The energy variance of the $\operatorname{MPS}|\Psi(\tilde{\mathcal{E}}, \tilde{\mathcal{F}})\rangle$ is measured by

$$
\begin{aligned}
\sigma^{2} & :=\frac{1}{N}\left(\left\langle\Psi(\tilde{\mathcal{E}}, \tilde{\mathcal{F}})\left|\hat{H}^{2}\right| \Psi(\tilde{\mathcal{E}}, \tilde{\mathcal{F}})\right\rangle-\langle\Psi(\tilde{\mathcal{E}}, \tilde{\mathcal{F}})|\hat{H}| \Psi(\tilde{\mathcal{E}}, \tilde{\mathcal{F}})\rangle^{2}\right) \\
& =\frac{1}{N}\left\langle\Psi(\tilde{\mathcal{E}}, \tilde{\mathcal{F}})\left|\hat{H}\left(1-\hat{P}_{\tilde{\mathcal{E}}, \tilde{\mathcal{F}}}\right) \hat{H}\right| \Psi(\tilde{\mathcal{E}}, \tilde{\mathcal{F}})\right\rangle,
\end{aligned}
$$

The quantum numbers are assigned by the following rule:

$$
n+m-1 / 2
$$$$
\uparrow
$$

$$
n \rightarrow \text { a nonzero block that creates } m \text { particles. }
$$

For example, the upper right block of $\mathcal{E}$ creates two particles, thus the block is labeled by $(0,3 / 2)$. For the product of $2 N$ matrices, we replace $1 / 2$ in the above rule with $2 N / 2=N$, such that

$$
\underbrace{\mathcal{E} \mathcal{F} \mathcal{E} \mathcal{F} \ldots \mathcal{E F}}_{2 N}=\begin{array}{l|c}
0 & 1 \\
1
\end{array}\left(\begin{array}{c|c}
\mathcal{X}_{11} & \mathcal{X}_{12} \\
\hline \mathcal{X}_{21} & \mathcal{X}_{22}
\end{array}\right),
$$

where $\mathcal{X}_{11}$ and $\mathcal{X}_{22}$ are $2 \times 2$ blocks which create $N$ particles, while $\mathcal{X}_{12}$ and $\mathcal{X}_{21}$ create $N+1$ and $N-1$ particles, respectively. In the thermodynamic limit $N \rightarrow \infty$, Eq. (E3) gives an MPS where the particle number equals the number of unit cells.

The exact ground state given by Eq. (E3) has bond dimension $\chi=4$. In general, however, we need to use an MPS with larger bond dimension to better approximate the true ground state. We can thus generalize Eq. (E1) to a block-banded form:

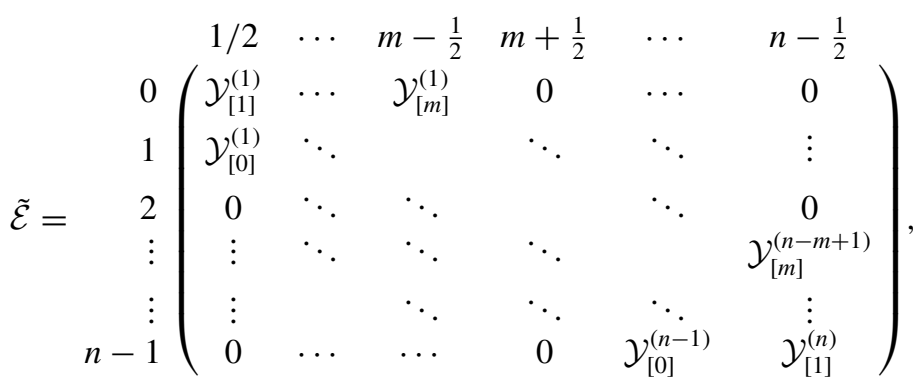

where $\hat{P}_{\tilde{\mathcal{E}}, \tilde{\mathcal{F}}}:=|\Psi(\tilde{\mathcal{E}}, \tilde{\mathcal{F}})\rangle\langle\Psi(\tilde{\mathcal{E}}, \tilde{\mathcal{F}})|$. Let

$$
\mathbb{H}_{r}^{\leqslant m}=\operatorname{span}\left(\left\{\prod_{\alpha=-1}^{1}\left(\hat{a}_{r, \alpha}^{\dagger}\right)^{n_{\alpha}}|\operatorname{vac}\rangle_{r} \mid \sum_{\alpha} n_{\alpha} \leqslant m\right\}\right)
$$

be the local truncated Hilbert space and $\hat{P}_{\forall \leqslant m}$ be the projection operator onto the total truncated Hilbert space $\mathbb{H}^{\forall \leqslant m}:=$ $\bigotimes_{i=r}^{2 N} \mathbb{H}_{r}^{\leqslant m}$. The Hamiltonian in $\mathbb{H}^{\forall \leqslant m}$ reads $\hat{H}_{\forall \leqslant m}:=$ $\hat{P}_{\forall \leqslant m} \hat{H} \hat{P}_{\forall \leqslant m}$. Equation (E5) can thus be rewritten as

$$
\begin{aligned}
\sigma^{2}= & \frac{1}{N}\left\langle\Psi(\tilde{\mathcal{E}}, \tilde{\mathcal{F}})\left|\hat{H}_{\forall \leqslant m}\left(1-\hat{P}_{\tilde{\mathcal{E}}, \tilde{\mathcal{F}}}\right) \hat{H}_{\forall \leqslant m}\right| \Psi(\tilde{\mathcal{E}}, \tilde{\mathcal{F}})\right\rangle \\
& +\frac{1}{N}\left\langle\Psi(\tilde{\mathcal{E}}, \tilde{\mathcal{F}})\left|\hat{H}\left(1-\hat{P}_{\forall \leqslant m}\right) \hat{H}\right| \Psi(\tilde{\mathcal{E}}, \tilde{\mathcal{F}})\right\rangle .
\end{aligned}
$$

The first term above can be viewed as the variance in $\mathbb{H}^{\forall \leqslant m}$, and it quantifies the effect of finite bond dimension. Similar to spin or fermion systems, the first term can be calculated 
TABLE VII. Examples of the actual values of the first and second terms in Eq. (E7) with $m=3, d=100, \lambda=1$, and $R=1$. The Haldane-critical phase transition happens between $\varphi=6 / 36$ and 7/36; see Fig. 2(b)

\begin{tabular}{lcc}
\hline \hline$\varphi / \pi$ & First & Second \\
\hline $1 / 36$ & $1.144 \times 10^{-4}$ & $8.082 \times 10^{-5}$ \\
$2 / 36$ & $3.463 \times 10^{-4}$ & $1.190 \times 10^{-4}$ \\
$3 / 36$ & $9.122 \times 10^{-4}$ & $1.267 \times 10^{-4}$ \\
$4 / 36$ & $1.957 \times 10^{-3}$ & $1.142 \times 10^{-4}$ \\
$5 / 36$ & $3.543 \times 10^{-3}$ & $9.620 \times 10^{-5}$ \\
$6 / 36$ & $5.702 \times 10^{-3}$ & $7.819 \times 10^{-5}$ \\
$7 / 36$ & $7.348 \times 10^{-3}$ & $6.284 \times 10^{-5}$ \\
$8 / 36$ & $8.614 \times 10^{-3}$ & $5.209 \times 10^{-5}$ \\
$9 / 36$ & $8.956 \times 10^{-3}$ & $4.874 \times 10^{-5}$ \\
\hline \hline
\end{tabular}

efficiently [50,113]. On the other hand, the second term quantifies the effect of truncation. Note that although $\hat{H}(1-$ $\left.\hat{P}_{\forall \leqslant m}\right) \hat{H}$ contains $\mathcal{O}\left(N^{2}\right)$ nonlocal terms, only $\mathcal{O}(N)$ local terms return nonzero values when sandwiched by $|\Psi(\tilde{\mathcal{E}}, \tilde{\mathcal{F}})\rangle$. Table VII shows that for $m=3$, at least near the Haldanecritical phase transition point, the effect of particle number truncation is about 100 times smaller than the effect of finite bond dimension.

Finally, we provide further numerical evidence that determines the shape of the phase boundary in the $\lambda=1$ plane in Fig. 2(a). The transfer matrix is defined as the sum of Kronecker products $\sum_{\tau, \tau^{\prime}} \tilde{E}^{\tau} \tilde{F}^{\tau^{\prime}} \otimes \tilde{E}^{\tau} \tilde{F}^{\tau^{\prime}}$, where $\tilde{E}^{\tau}:=$ $\langle\tau|\tilde{\mathcal{E}}|$ vac $\rangle$ and $\tilde{F}^{\tau}:=\langle\tau|\tilde{\mathcal{F}}|$ vac $\rangle$ with $\{|\tau\rangle\}$ being a basis in the Fock space. Let $\epsilon_{i}=-\ln \left|\lambda_{i}\right|$, where $\lambda_{i}$ is the $i$ th largest absolute eigenvalue of the transfer matrix, and $\left|\lambda_{1}\right|$ is normalized to 1 . As we change the bond dimension $\chi$, we calculate the scaling of the inverse correlation length $1 / \xi:=\epsilon_{2}$ with respect to $\epsilon_{3}-\epsilon_{2}$ along the path parameterized by $(R \sin \varphi, R \cos \varphi, 1)$; see Fig. 14. We find that, as $R$ grows, the phase transition occurs at smaller $\varphi$, which indicates that the phase boundary is curved instead of straight.

\section{APPENDIX F: UNIQUENESS OF THE GROUND STATE OF $\hat{H}^{f, X}$}

Mathematically, the uniqueness of the ground state of $\hat{H}^{f, X}$ can be proved with additional assumptions: $\Lambda_{X}^{[1]} \neq \varnothing$ and $g_{S, \boldsymbol{r}}>0$ for $\forall S$ and $\forall \boldsymbol{r} \in \Lambda_{X}^{[1]}$. With the "completeness relation" $\sum_{S} \hat{P}_{r}^{(S)}=\hat{n}_{r}\left(\hat{n}_{r}-1\right) / 2$ in mind and following the deduction in Eq. (10), one can show that the ground state can only be a linear combination of FPSs. The uniqueness of the ground state of $\hat{H}^{f, X}$ then follows from the uniqueness of $\left|\mathrm{VBS}_{f, X^{\prime}}\right\rangle$. The assumption $\Lambda_{X}^{[1]} \neq \emptyset$ is always satisfied in lattices generated by the cell construction; see, for example, Fig. 4(b) and Fig. 5(a). However, for the kagome lattice shown in Fig. 5(b), $\Lambda_{X}^{[1]}=\emptyset$. Nevertheless, we propose the following conjecture: even in lattice $X$ with $\Lambda_{X}^{[1]}=\emptyset$, the exact ground state of $\hat{H}_{X}$ is unique when $X^{\prime}$ is not a bipartite lattice. For the kagome lattice, $X^{\prime}$ is a triangular lattice which is not bipartite. Note that if $X^{\prime}$ is bipartite and $\Lambda_{X}^{[1]}=\emptyset$, the ground state of $\hat{H}^{f, X}$ will be degenerate. For example, for the Creutz ladder in
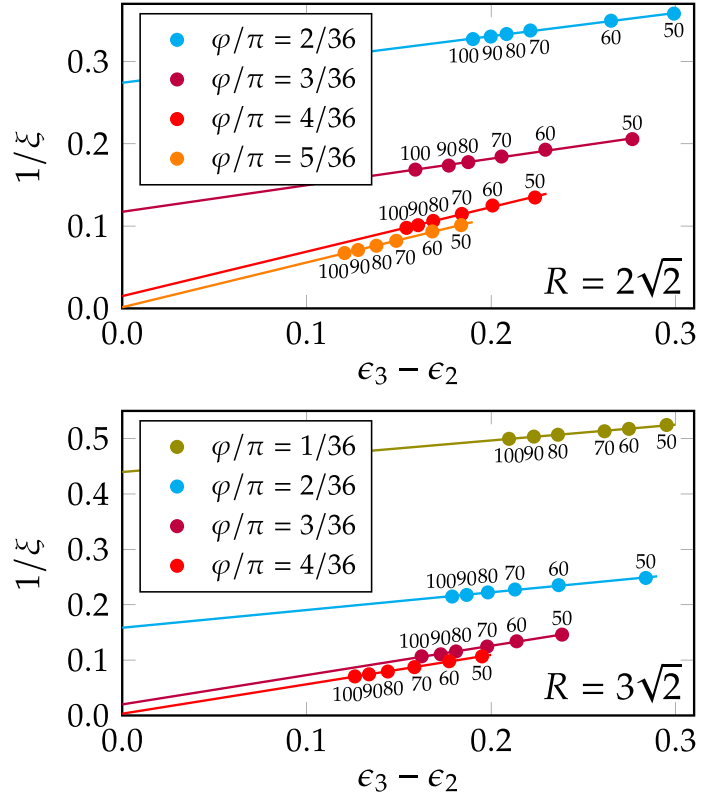

FIG. 14. Scaling of the inverse correlation length $1 / \xi:=\epsilon_{2}$ with respect to $\epsilon_{3}-\epsilon_{2}$. Numbers near the data points denote the corresponding bond dimensions $d$ of each block. Along the path with $R=2 \sqrt{2}$, we see that a quantum phase transition occurs between $\varphi=4 \pi / 36$ and $5 \pi / 36$. On the other hand, along the path with $R=3 \sqrt{2}$, a phase transition occurs between $\varphi=3 \pi / 36$ and $4 \pi / 36$.

(a)

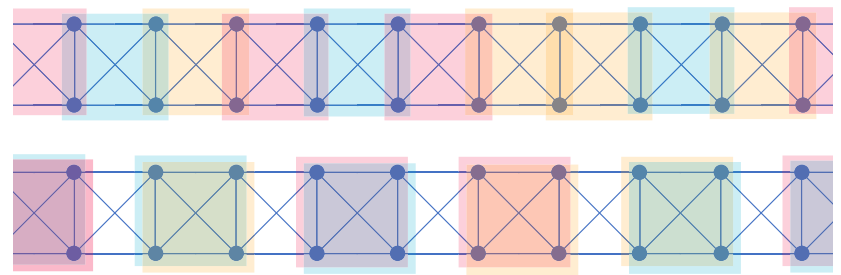

(b)
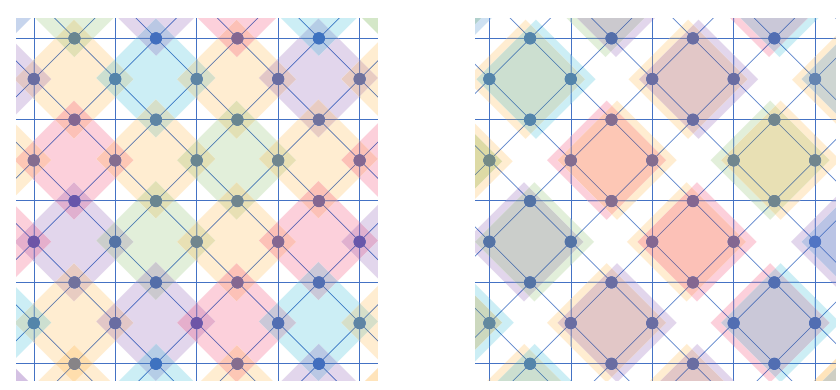

FIG. 15. A "nontrivial" ground state is degenerated with product states, if $\Lambda_{X}^{[1]}=\varnothing$ and the corresponding quantum spin model lives on a bipartite lattice. (a) Spin-1 bosons on a Creutz ladder. Each colored square denotes a CLS with spin degree of freedom. Note that the ground stats are superpositions of all allowed spin configurations. (b) Spin-2 bosons on a checkerboard lattice. $X^{\prime}$ in this case is a square lattice. 
(a)

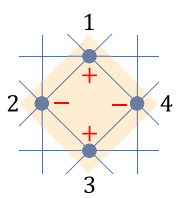

(b)

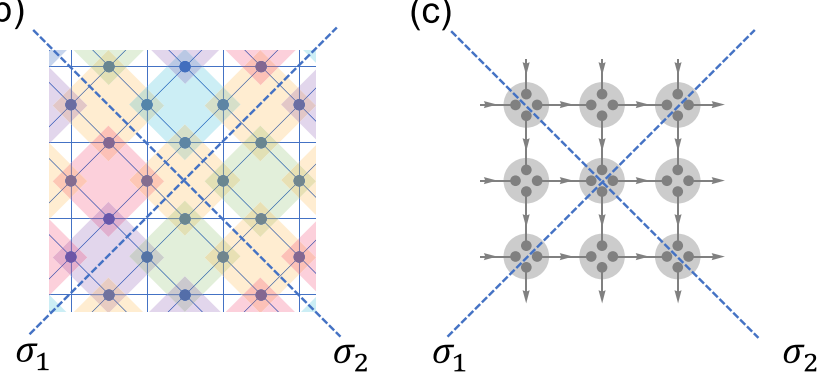

(d) (a)

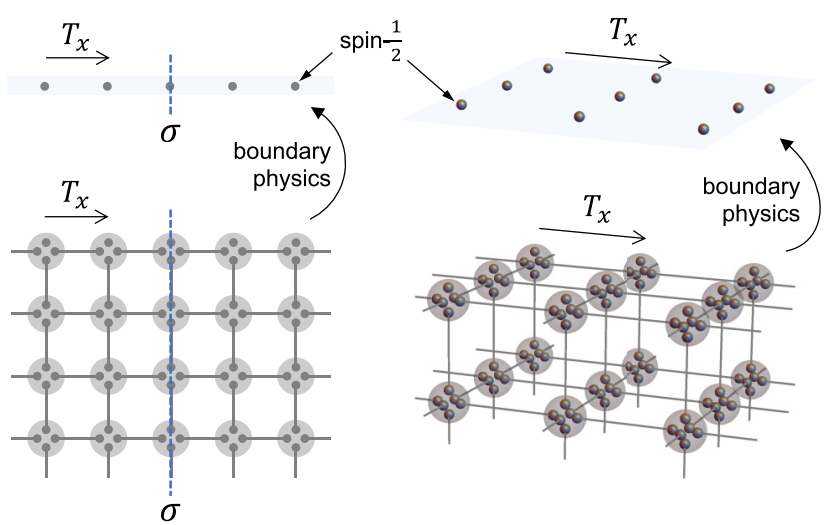

FIG. 17. LSM theorems as a special case of constraints at the boundaries of SPT phases: a gapless or degenerate edge state ensured by certain symmetry in the boundary implies an SPT phase protected by the same symmetry in the bulk. The translation group trn is generated by $T_{x}$, while the group $D_{1}$ is generated by the site-centered reflection $\sigma$. (a) A spin- $1 / 2$ simple linear chain can be regarded as the edge of $\left|\mathrm{VBS}_{2, \square}\right\rangle$. (b) A spin-1/2 system on a square lattice can be regarded as the surface of $\left|\mathrm{VBS}_{3, \mathbb{E}}\right\rangle$.

FIG. 16. (a) A CLS in the checkerboard lattice. Sign of the amplitude alternates from site 1 to 4. (b) An FPS on the checkerboard lattice. $\left(\left|\mathrm{GS}_{2, \bigotimes}\right\rangle\right.$ is $D_{2}$ symmetric, and it is a superposition of FPSs with different spin configurations.) We require that the symmetry center of $D_{2}$ lies at the geometric center of a CLS and the two mirror planes are placed as in the figure. (c) For $\left|\mathrm{VBS}_{2, \square}\right\rangle$, the symmetry center should lie at a site (spin) in order to be compatible with (b). (d) $1 \mathrm{D}$ representations of $D_{2}$ associated with $\left|\mathrm{GS}_{2, \bigotimes}\right\rangle$ and $\left|\mathrm{VBS}_{2, \square}\right\rangle$. The former state yields a nontrivial representation and is thus in an SPT phase.

Fig. 15(a); let $\left(\hat{B}_{j, \beta_{j}}^{1, \bigotimes}\right)^{\dagger}$ create a CLS of spin-1 boson, it is easy to see that the following two states both have zero energy:

$$
\begin{gathered}
\operatorname{Tr} \prod_{j=1}^{N}\left[\sum_{\beta_{j}} M^{\beta_{j}}\left(\hat{B}_{j, \beta_{j}}^{1, \bigotimes}\right)^{\dagger}\right]|\mathrm{vac}\rangle, \\
\prod_{\ell=1}^{N / 2}\left[\left(\hat{B}_{2 \ell, 0}^{1, \bigotimes}\right)^{\dagger}\left(\hat{B}_{2 \ell, 0}^{1, \bigotimes}\right)^{\dagger}-2\left(\hat{B}_{2 \ell, 1}^{1, \bigotimes}\right)^{\dagger}\left(\hat{B}_{2 \ell,-1}^{1, \bigotimes}\right)^{\dagger}\right]|\mathrm{vac}\rangle .
\end{gathered}
$$

These two states are depicted in Fig. 15(a). The first "nontrivial" state is a linear combination of FPSs, while the second state is a product state. A similar thing happens in spin-2 bosons loaded on the checkerboard lattice; see Fig. 15(b). In such cases, though the Hamiltonians $\hat{H}^{f, X}$ do not exhibit any nontrivial phases due to the degeneracy, the "nontrivial" ground state (= the state which is a linear combination of FPSs) can always be regarded as the unique ground states of some other (usually more complicated) parent Hamiltonians [70], and hence the classification of such "nontrivial" states from the viewpoint of SPT phases still makes sense. For example, let $\left|\mathrm{GS}_{2, \bigotimes}\right\rangle$ be the "nontrivial" ground state of spin-2 bosons on the checkerboard lattice. Following the discussion in Sec. III C, by properly chosing the symmetry center and the mirror planes, one can show that $\left|\mathrm{GS}_{2, \mathbb{} \text { ( })}\right\rangle$ is in an SPT phase protected by $D_{2}$; see Fig. 16 .

\section{APPENDIX G: LIEB-SCHULTZ-MATTIS (LSM) THEOREMS AND SPT PHASES}

LSM theorems are a class of no-go theorems on the "ingappability" of certain quantum systems. These theorems ensure that a spin system with a half-odd-integer spin per unit cell can never have a unique gapped ground state, if certain on-site $\times$ spatial symmetry is present. The original LSM theorem $[114,115]$ is about $1 \mathrm{D}$ systems with the combination of $\mathrm{SO}(3)$ and translation symmetry [denote the symmetry group as $\mathrm{SO}(3) \times \operatorname{trn}]$. The theorem is then extended to higher dimensions [116-118].

Recently, more symmetries other than $\mathrm{SO}(3) \times$ trn have been found to render the ingappability [66,119-125]. For example, it is now known that the combination of timereversal (TR) and site-centered reflection symmetry (denote the symmetry group as $\mathrm{TR} \times D_{1}$ ) in $1 \mathrm{D}$ systems with a half-odd-integer spin per unit cell also guarantees the ingappability $[119,122,123]$. Other such symmetries in one dimension include $\mathbb{Z}_{2} \times \mathbb{Z}_{2} \times \operatorname{trn}, \mathbb{Z}_{2} \times \mathbb{Z}_{2} \times D_{1}$, TR $\times$ trn, and so on $[119,122,123]$. In fact, $\mathbb{Z}_{2} \times \mathbb{Z}_{2} \times$ trn and TR $\times$ trn apply to any $d \geqslant 1$ dimensions [124].

The boundary (edge state) of an SPT phase is usually gapless or degenerate [126], coincident with the statements of the LSM theorems. Indeed, the LSM theorems are, in a precise sense, a special case of constraints at the boundaries of SPT phases [127]. In other words, a gapless or degenerate edge state ensured by certain symmetry in the boundary implies an SPT phase protected by the same symmetry in the bulk, which is known as the bulk-boundary correspondence. For example, a spin- $1 / 2$ simple linear chain can be thought of as the edge of $\left|\mathrm{VBS}_{2, \square}\right\rangle$, the spin-2 VBS state on a square lattice. Similarly, a spin-1/2 system on a square lattice can be regarded as the surface of $\left|\mathrm{VBS}_{3, \mathbb{E}}\right\rangle$, the spin-3 VBS state on a cubic lattice; 
see Fig. 17. The ingappability of a spin- $1 / 2$ simple linear chain due to the TR $\times D_{1}$ or $\mathbb{Z}_{2} \times \mathbb{Z}_{2} \times D_{1}$ symmetry implies that $\left|\mathrm{VBS}_{2, \square}\right\rangle$ is in an SPT phase protected by TR $\times D_{1}$ or $\mathbb{Z}_{2} \times \mathbb{Z}_{2} \times D_{1}$. Similarly, one can also show that $\left|\mathrm{VBS}_{2, \square}\right\rangle$ and $\left|\mathrm{VBS}_{3, \mathbb{E}}\right\rangle$ are in an SPT phase protected by $\mathbb{Z}_{2} \times \mathbb{Z}_{2} \times$ trn and TR $\times$ trn, as summarized in Table V. Note that the edge of $\left|\mathrm{VBS}_{3, \Delta}\right\rangle$ is a spin-1 chain, so that the LSM theorems do not apply.
[1] B. Zeng, X. Chen, D.-L. Zhou, and X.-G. Wen, Quantum Information Meets Quantum Matter (Springer, New York, 2019).

[2] I. Affleck, T. Kennedy, E. H. Lieb, and H. Tasaki, Rigorous Results on Valence-Bond Ground States in Antiferromagnets, Phys. Rev. Lett. 59, 799 (1987).

[3] I. Affleck, T. Kennedy, E. H. Lieb, and H. Tasaki, Valence bond ground states in isotropic quantum antiferromagnets, Commun. Math. Phys. 115, 477 (1988).

[4] F. Pollmann, A. M. Turner, E. Berg, and M. Oshikawa, Entanglement spectrum of a topological phase in one dimension, Phys. Rev. B 81, 064439 (2010).

[5] F. Pollmann, E. Berg, A. M. Turner, and M. Oshikawa, Symmetry protection of topological phases in one-dimensional quantum spin systems, Phys. Rev. B 85, 075125 (2012).

[6] Z.-C. Gu and X.-G. Wen, Tensor-entanglement-filtering renormalization approach and symmetry-protected topological order, Phys. Rev. B 80, 155131 (2009).

[7] H. Tasaki, Physics and Mathematics of Quantum Many-Body Systems (Springer, New York, 2020).

[8] T. Kennedy and H. Tasaki, Hidden $Z_{2} \times Z_{2}$ symmetry breaking in Haldane-gap antiferromagnets, Phys. Rev. B 45, 304 (1992).

[9] M. Oshikawa, Hidden $Z_{2} \times Z_{2}$ symmetry in quantum spin chains with arbitrary integer spin, J. Phys. Condens. Matter 4, 7469 (1992).

[10] T. A. Hilker, G. Salomon, F. Grusdt, A. Omran, M. Boll, E. Demler, I. Bloch, and C. Gross, Revealing hidden antiferromagnetic correlations in doped Hubbard chains via string correlators, Science 357, 484 (2017).

[11] S. de Léséleuc, V. Lienhard, P. Scholl, D. Barredo, S. Weber, N. Lang, H. P. Büchler, T. Lahaye, and A. Browaeys, Observation of a symmetry-protected topological phase of interacting bosons with Rydberg atoms, Science 365, 775 (2019).

[12] E. G. Dalla Torre, E. Berg, and E. Altman, Hidden Order in 1D Bose Insulators, Phys. Rev. Lett. 97, 260401 (2006).

[13] E. Berg, E. G. Dalla Torre, T. Giamarchi, and E. Altman, Rise and fall of hidden string order of lattice bosons, Phys. Rev. B 77, 245119 (2008).

[14] D. Rossini and R. Fazio, Phase diagram of the extended BoseHubbard model, New J. Phys. 14, 065012 (2012).

[15] J. Xu, Q. Gu, and E. J. Mueller, Realizing the Haldane Phase with Bosons in Optical Lattices, Phys. Rev. Lett. 120, 085301 (2018).

[16] G. G. Batrouni, R. T. Scalettar, V. G. Rousseau, and B. Grémaud, Competing Supersolid and Haldane Insulator Phases in the Extended One-Dimensional Bosonic Hubbard Model, Phys. Rev. Lett. 110, 265303 (2013).

[17] S. Ejima, F. Lange, and H. Fehske, Spectral and Entanglement Properties of the Bosonic Haldane Insulator, Phys. Rev. Lett. 113, 020401 (2014).

[18] F. Lange, S. Ejima, and H. Fehske, Anyonic Haldane Insulator in One Dimension, Phys. Rev. Lett. 118, 120401 (2017).
[19] M. Dalmonte, M. Di Dio, L. Barbiero, and F. Ortolani, Homogeneous and inhomogeneous magnetic phases of constrained dipolar bosons, Phys. Rev. B 83, 155110 (2011).

[20] K. Sugimoto, S. Ejima, F. Lange, and H. Fehske, Quantum phase transitions in the dimerized extended Bose-Hubbard model, Phys. Rev. A 99, 012122 (2019).

[21] J. Zhao, S. Hu, and P. Zhang, Symmetry-Protected Topological Phase in a One-Dimensional Correlated Bosonic Model with a Synthetic Spin-Orbit Coupling, Phys. Rev. Lett. 115, 195302 (2015).

[22] B. Grémaud and G. G. Batrouni, Haldane phase on the sawtooth lattice: Edge states, entanglement spectrum, and the flat band, Phys. Rev. B 95, 165131 (2017).

[23] J. J. García-Ripoll, M. A. Martin-Delgado, and J. I. Cirac, Implementation of Spin Hamiltonians in Optical Lattices, Phys. Rev. Lett. 93, 250405 (2004).

[24] H. Nonne, P. Lecheminant, S. Capponi, G. Roux, and E. Boulat, Haldane charge conjecture in one-dimensional multicomponent fermionic cold atoms, Phys. Rev. B 81, 020408(R) (2010).

[25] V. Bois, S. Capponi, P. Lecheminant, M. Moliner, and K. Totsuka, Phase diagrams of one-dimensional half-filled twoorbital SU( $n)$ cold fermion systems, Phys. Rev. B 91, 075121 (2015).

[26] S. Moudgalya and F. Pollmann, Fragility of symmetryprotected topological order on a Hubbard ladder, Phys. Rev. B 91, 155128 (2015).

[27] F. Lange, S. Ejima, and H. Fehske, Criticality at the Haldaneinsulator charge-density-wave quantum phase transition, Phys. Rev. B 92, 041120(R) (2015).

[28] M. Nakagawa and N. Kawakami, Symmetry-protected topological phase transition in one-dimensional Kondo lattice and its realization with ultracold atoms, Phys. Rev. B 96, 155133 (2017).

[29] H. Ueda, T. Morimoto, and T. Momoi, Symmetry protected topological phases in two-orbital SU(4) fermionic atoms, Phys. Rev. B 98, 045128 (2018).

[30] P. Fromholz, S. Capponi, P. Lecheminant, D. J. Papoular, and K. Totsuka, Haldane phases with ultracold fermionic atoms in double-well optical lattices, Phys. Rev. B 99, 054414 (2019).

[31] S. Fazzini, L. Barbiero, and A. Montorsi, Interaction-Induced Fractionalization and Topological Superconductivity in the Polar Molecules Anisotropic $t-J$ Model, Phys. Rev. Lett. 122, 106402 (2019).

[32] A. Montorsi, S. Fazzini, and L. Barbiero, Homogeneous and domain-wall topological Haldane conductors with dressed Rydberg atoms, Phys. Rev. A 101, 043618 (2020).

[33] Y. Kawaguchi and M. Ueda, Spinor Bose-Einstein condensates, Phys. Rep. 520, 253 (2012); D. M. Stamper-Kurn and M. Ueda, Spinor Bose gases: Symmetries, magnetism, and quantum dynamics, Rev. Mod. Phys. 85, 1191 (2013). 
[34] H. Katsura and H. Tasaki, Ground States of the Spin-1 BoseHubbard Model, Phys. Rev. Lett. 110, 130405 (2013).

[35] H. Yang and H. Katsura, Rigorous Results for the Ground States of the Spin-2 Bose-Hubbard Model, Phys. Rev. Lett. 122, 053401 (2019).

[36] A. Läuchli, G. Schmid, and S. Trebst, Spin nematics correlations in bilinear-biquadratic $S=1$ spin chains, Phys. Rev. B 74, 144426 (2006).

[37] A. Eckardt, C. Weiss, and M. Holthaus, Superfluid-Insulator Transition in a Periodically Driven Optical Lattice, Phys. Rev. Lett. 95, 260404 (2005).

[38] A. Eckardt, P. Hauke, P. Soltan-Panahi, C. Becker, K. Sengstock, and M. Lewenstein, Frustrated quantum antiferromagnetism with ultracold bosons in a triangular lattice, Europhys. Lett. 89, 10010 (2010).

[39] D. H. Dunlap and V. M. Kenkre, Dynamic localization of a charged particle moving under the influence of an electric field, Phys. Rev. B 34, 3625 (1986).

[40] F. Grossmann, T. Dittrich, P. Jung, and P. Hänggi, Coherent Destruction of Tunneling, Phys. Rev. Lett. 67, 516 (1991).

[41] F. Großmann and P. Hänggi, Localization in a driven two-level dynamics, Europhys. Lett. 18, 571 (1992).

[42] K. Drese and M. Holthaus, Exploring a Metal-Insulator Transition with Ultracold Atoms in Standing Light Waves?, Phys. Rev. Lett. 78, 2932 (1997).

[43] H. Lignier, C. Sias, D. Ciampini, Y. Singh, A. Zenesini, O. Morsch, and E. Arimondo, Dynamical Control of Matter-Wave Tunneling in Periodic Potentials, Phys. Rev. Lett. 99, 220403 (2007).

[44] A. Eckardt, M. Holthaus, H. Lignier, A. Zenesini, D. Ciampini, O. Morsch, and E. Arimondo, Exploring dynamic localization with a Bose-Einstein condensate, Phys. Rev. A 79, 013611 (2009).

[45] E. Kierig, U. Schnorrberger, A. Schietinger, J. Tomkovic, and M. K. Oberthaler, Single-Particle Tunneling in Strongly Driven Double-Well Potentials, Phys. Rev. Lett. 100, 190405 (2008).

[46] A. Zenesini, H. Lignier, D. Ciampini, O. Morsch, and E. Arimondo, Coherent Control of Dressed Matter Waves, Phys. Rev. Lett. 102, 100403 (2009).

[47] J. Struck, C. Ölschläger, R. Le Targat, P. Soltan-Panahi, A. Eckardt, M. Lewenstein, P. Windpassinger, and K. Sengstock, Quantum simulation of frustrated classical magnetism in triangular optical lattices, Science 333, 996 (2011).

[48] J.-W. Rhim and B.-J. Yang, Classification of flat bands according to the band-crossing singularity of Bloch wave functions, Phys. Rev. B 99, 045107 (2019).

[49] N. Read, Compactly supported Wannier functions and algebraic $k$-theory, Phys. Rev. B 95, 115309 (2017).

[50] V. Zauner-Stauber, L. Vanderstraeten, M. T. Fishman, F. Verstraete, and J. Haegeman, Variational optimization algorithms for uniform matrix product states, Phys. Rev. B 97, 045145 (2018).

[51] L. Vanderstraeten, J. Haegeman, and F. Verstraete, Tangent-space methods for uniform matrix product states, SciPost Phys. Lect. Notes, 7 (2019), doi: 10.21468/SciPostPhysLectNotes.7.

[52] A. Imambekov, M. Lukin, and E. Demler, Spin-exchange interactions of spin-one bosons in optical lattices: Singlet, nematic, and dimerized phases, Phys. Rev. A 68, 063602 (2003).
[53] S. Tsuchiya, S. Kurihara, and T. Kimura, Superfluid-Mott insulator transition of spin-1 bosons in an optical lattice, Phys. Rev. A 70, 043628 (2004).

[54] T.-L. Ho, Spinor Bose Condensates in Optical Traps, Phys. Rev. Lett. 81, 742 (1998); T. Ohmi and K. Machida, BoseEinstein condensation with internal degrees of freedom in alkali atom gases, J. Phys. Soc. Jpn. 67, 1822 (1998).

[55] H. Tasaki, Ferromagnetism in the Hubbard Models with Degenerate Single-Electron Ground States, Phys. Rev. Lett. 69, 1608 (1992).

[56] H. Tasaki, From Nagaoka's ferromagnetism to flat-band ferromagnetism and beyond: An introduction to ferromagnetism in the Hubbard model, Prog. Theor. Phys. 99, 489 (1998).

[57] K. Tamura and H. Katsura, Ferromagnetism in the SU( $n$ ) Hubbard model with a nearly flat band, Phys. Rev. B 100, 214423 (2019).

[58] T. Zhang and G.-B. Jo, One-dimensional sawtooth and zigzag lattices for ultracold atoms, Sci. Rep. 5, 16044 (2015).

[59] J. B. Parkinson, On the integrability of the $S=1$ quantum spin chain with pure biquadratic exchange, J. Phys. C 20, L1029 (1987).

[60] J. B. Parkinson, The $S=1$ quantum spin chain with pure biquadratic exchange, J. Phys. C 21, 3793 (1988).

[61] A. Mielke and H. Tasaki, Ferromagnetism in the Hubbard model. Examples from models with degenerate single-electron ground states, Comm. Math. Phys. 158, 341 (1993).

[62] One can verify that the largest absolute eigenvalue of the transfer matrix $\sum_{\tau, \tau^{\prime}} F^{\tau} E^{\tau^{\prime}} \otimes F^{\tau} E^{\tau^{\prime}}$ is nondegenerate. This is equivalent to the statement that the MPS in Eq. (19) is injective.

[63] Y.-T. Oh, H. Katsura, H.-Y. Lee, and J. H. Han, Proposal of a spin-one chain model with competing dimer and trimer interactions, Phys. Rev. B 96, 165126 (2017).

[64] M. Andres, I. Schneider, and S. Eggert, Highest weight state description of the isotropic spin-1 chain, Phys. Rev. B 77, 014429 (2008).

[65] Equation (22) is usually proved in the canonical form; see Ref. [128] or Theorem 7 in Ref. [129]. However, the equation holds regardless of the form of an injective MPS; see Ref. [7] or Sec. 7.3 in Ref. [130].

[66] X. Chen, Z.-C. Gu, and X.-G. Wen, Classification of gapped symmetric phases in one-dimensional spin systems, Phys. Rev. B 83, 035107 (2011).

[67] F. Pollmann and A. M. Turner, Detection of symmetryprotected topological phases in one dimension, Phys. Rev. B 86, 125441 (2012)

[68] Y. Fuji, F. Pollmann, and M. Oshikawa, Distinct Trivial Phases Protected by a Point-Group Symmetry in Quantum Spin Chains, Phys. Rev. Lett. 114, 177204 (2015).

[69] H. Song, S.-J. Huang, L. Fu, and M. Hermele, Topological Phases Protected by Point Group Symmetry, Phys. Rev. X 7, 011020 (2017).

[70] D. Perez-Garcia, F. Verstraete, J. I. Cirac, and M. M. Wolf, PEPS as unique ground states of local Hamiltonians, Quantum Inf. Comput. 8, 0650 (2008)

[71] Z.-X. Liu, M. Liu, and X.-G. Wen, Gapped quantum phases for the $S=1$ spin chain with $D_{2 h}$ symmetry, Phys. Rev. B 84, 075135 (2011).

[72] V. Zauner-Stauber, L. Vanderstraeten, J. Haegeman, I. P. McCulloch, and F. Verstraete, Topological nature of spinons 
and holons: Elementary excitations from matrix product states with conserved symmetries, Phys. Rev. B 97, 235155 (2018).

[73] M. M. Rams, P. Czarnik, and L. Cincio, Precise Extrapolation of the Correlation Function Asymptotics in Uniform Tensor Network States with Application to the Bose-Hubbard and XXZ Models, Phys. Rev. X 8, 041033 (2018).

[74] L. Tagliacozzo, T. R. de Oliveira, S. Iblisdir, and J. I. Latorre, Scaling of entanglement support for matrix product states, Phys. Rev. B 78, 024410 (2008).

[75] S. R. Manmana, A. M. Läuchli, F. H. L. Essler, and F. Mila, Phase diagram and continuous pair-unbinding transition of the bilinear-biquadratic $S=1$ Heisenberg chain in a magnetic field, Phys. Rev. B 83, 184433 (2011).

[76] A. N. Kirillov and V. E. Korepin, The valence bond solid in quasicrystals, Leningrad Math. J. 1, 343 (1990).

[77] H. Katsura, N. Kawashima, A. N. Kirillov, V. E. Korepin, and S. Tanaka, Entanglement in valence-bond-solid states on symmetric graphs, J. Phys. A 43, 255303 (2010).

[78] T. Kennedy, E. H. Lieb, and H. Tasaki, A two-dimensional isotropic quantum antiferromagnet with unique disordered ground state, J. Stat. Phys. 53, 383 (1988).

[79] A. Mielke, Ferromagnetism in the Hubbard model and Hund's rule, Phys. Lett. A 174, 443 (1993).

[80] A. Mielke, Exact ground states for the Hubbard model on the kagome lattice, J. Phys. A 25, 4335 (1992).

[81] A. Mielke, Ferromagnetism in the Hubbard model on line graphs and further considerations, J. Phys. A 24, 3311 (1991).

[82] L. Morales-Inostroza and R. A. Vicencio, Simple method to construct flat-band lattices, Phys. Rev. A 94, 043831 (2016).

[83] W. Maimaiti, A. Andreanov, H. C. Park, O. Gendelman, and $\mathrm{S}$. Flach, Compact localized states and flat-band generators in one dimension, Phys. Rev. B 95, 115135 (2017).

[84] R. Dias and J. Gouveia, Origami rules for the construction of localized eigenstates of the Hubbard model in decorated lattices, Sci. Rep. 5, 16852 (2015).

[85] A. Tanaka, An extension of the cell-construction method for the flat-band ferromagnetism, J. Stat. Phys. 181, 897(2020).

[86] Z. Liu, F. Liu, and Y.-S. Wu, Exotic electronic states in the world of flat bands: From theory to material, Chin. Phys. B 23, 077308 (2014).

[87] In fact, the sawtooth chain can be produced by either the cell construction or the line graph construction.

[88] Z. Gulácsi, A. Kampf, and D. Vollhardt, Exact Many-Electron Ground States on the Diamond Hubbard Chain, Phys. Rev. Lett. 99, 026404 (2007).

[89] G.-B. Jo, J. Guzman, C. K. Thomas, P. Hosur, A. Vishwanath, and D. M. Stamper-Kurn, Ultracold Atoms in a Tunable Optical Kagome Lattice, Phys. Rev. Lett. 108, 045305 (2012).

[90] Ignore spin for the moment, i.e., take $f=0$. In $d=1, N$ different CLSs can always be chosen to be linearly independent. However, in $d>1$ with PBC, these $N$ CLSs can be linearly dependent in some cases, such as in kagome lattice [48]. Nevertheless, they can still be linearly independent in $d>1$ with $\mathrm{OBC}$.

[91] "On-site symmetry" is also called "internal symmetry." It refers to a global symmetry that can be factorized siteby-site, and the symmetry operation on each site is an endomorphism of the on-site Hilbert space. The $\mathbb{Z}_{2} \times \mathbb{Z}_{2}$ spin rotation is an on-site symmetry because, for example, $\hat{U}(z)=$ $\prod_{r} \exp \left(-\mathrm{i} \pi \hat{S}_{r}^{z}\right)$, and $\hat{S}_{r}^{z}$ acts only on the local Hilbert space.
[92] See Sec. IIIC3 or Refs. [1,127,131-133]. When the translation symmetry is indispensable to protect an SPT phase, such a phase is often called a weak SPT phase.

[93] Consider $\left|\mathrm{VBS}_{3, \Delta}\right\rangle$ defined on a half-infinite plane. On its 1D boundary, every site hosts two "dangling" spin-1/2's, as

shown in the figure: $\%$. Since the six spin-1/2's on the same site form a totally symmetric spin-3 degree of freedom, the two "dangling" spin-1/2's have to form a symmetric spin-1 degree of freedom. One can add perturbations at the boundary that couple these spin-1's through, for example, the translation invariant spin-1 AKLT Hamiltonian. This perturbation thus results in a gapped edge state without breaking the combination of $\mathrm{SO}(3)$ and translation symmetry, and hence the state $\left|\mathrm{VBS}_{3, \Delta}\right\rangle$ is in a trivial phase protected by such symmetry. In this sense, we say that the edge state of $\left|\mathrm{VBS}_{3, \Delta}\right\rangle$ can be trivially gapped out. As for $\left|\mathrm{VBS}_{2, \square}\right\rangle$, its edge state cannot be gapped out while preserving the $\mathrm{SO}(3) \times$ trn symmetry; see Appendix G or Refs. [1,132,133].

[94] All 1D representations of $G$ form an Abelian group, which is the first cohomology group $H^{1}[G, U(1)]$. In $d=1,2$ dimensions, $H^{1}[G, U(1)]$ is believed to give a complete classification of phases protected by the point group $G$. In $d=3$ dimension, extra indices are needed for a complete classification. See Refs. $[69,95,96]$ for general classification theories.

[95] S.-J. Huang, H. Song, Y.-P. Huang, and M. Hermele, Building crystalline topological phases from lower-dimensional states, Phys. Rev. B 96, 205106 (2017).

[96] R. Thorngren and D. V. Else, Gauging Spatial Symmetries and the Classification of Topological Crystalline Phases, Phys. Rev. X 8, 011040 (2018).

[97] In the thermodynamic limit, it may be subtle to ask if the number is even or odd. Nevertheless, it is always possible to identify the phase of a finite-size system. The fact is that the symmetry-protected phase of a finite-size system should be identical to that of an infinite system. The reason is as follows. All the symmetry-protected phases are about local properties of the system, since there is only short-range entanglement in the bulk [1]. In fact, according to Refs. [69,95], the pointgroup-symmetry-protected phases of a 1D or 2D system are determined by the properties of a local region around the symmetry center, and the size of the local region roughly agrees with the correlation length. Local properties are obviously not affected by the those degrees of freedom that are infinitely far away.

[98] How can the same point group in the same system results in two distinct phases by only choosing a different symmetry center? The reason is that the plaquette-centered symmetry and the bond-centered symmetry are inequivalent in the sense that one symmetry alone does not imply the other. In the presence of translation symmetry, point groups centered in inequivalent positions are included in a larger space group (or wallpaper group). It is reasonable to say that the state $\left|\mathrm{VBS}_{2, \square}\right\rangle$ is in an SPT phase protected by the wallpaper group $p 4 m$, since bond-centered $D_{2}$ is a subgroup of $p 4 m$. See Ref. [95] for the theory of wallpaper-group-protected phases.

[99] The state in Eq. (19) with $0<|\lambda|<\infty$ is inversion symmetric only about a site. In Table I we can see that this path smoothly connects two distinct phases even when the (site-centered) inversion symmetry is preserved. 
[100] H. Yao and S. A. Kivelson, Fragile Mott Insulators, Phys. Rev. Lett. 105, 166402 (2010).

[101] S. A. Parameswaran, I. Kimchi, A. M. Turner, D. M. Stamper-Kurn, and A. Vishwanath, Wannier Permanent Wave Functions for Featureless Bosonic Mott Insulators on the 1/3-Filled Kagome Lattice, Phys. Rev. Lett. 110, 125301 (2013).

[102] I. Kimchi, S. Parameswaran, A. M. Turner, F. Wang, and A. Vishwanath, Featureless and nonfractionalized Mott insulators on the honeycomb lattice at $1 / 2$ site filling, Proc. Natl. Acad. Sci. USA 110, 16378 (2013).

[103] A. Griesmaier, J. Werner, S. Hensler, J. Stuhler, and T. Pfau, Bose-Einstein Condensation of Chromium, Phys. Rev. Lett. 94, 160401 (2005).

[104] M. Lu, N. Q. Burdick, S. H. Youn, and B. L. Lev, Strongly Dipolar Bose-Einstein Condensate of Dysprosium, Phys. Rev. Lett. 107, 190401 (2011).

[105] K. Aikawa, A. Frisch, M. Mark, S. Baier, A. Rietzler, R. Grimm, and F. Ferlaino, Bose-Einstein Condensation of Erbium, Phys. Rev. Lett. 108, 210401 (2012).

[106] Y. Kawaguchi, H. Saito, and M. Ueda, Einstein-De Haas Effect in Dipolar Bose-Einstein Condensates, Phys. Rev. Lett. 96, 080405 (2006).

[107] Y. Cao, V. Fatemi, S. Fang, K. Watanabe, T. Taniguchi, E. Kaxiras, and P. Jarillo-Herrero, Unconventional superconductivity in magic-angle graphene superlattices, Nature (London) 556, 43 (2018).

[108] C. J. Turner, A. A. Michailidis, D. A. Abanin, M. Serbyn, and Z. Papić, Weak ergodicity breaking from quantum many-body scars, Nat. Phys. 14, 745 (2018).

[109] W. W. Ho, S. Choi, H. Pichler, and M. D. Lukin, Periodic Orbits, Entanglement, and Quantum Many-Body Scars in Constrained Models: Matrix Product State Approach, Phys. Rev. Lett. 122, 040603 (2019).

[110] Y. Kuno, T. Mizoguchi, and Y. Hatsugai, Flat band quantum scar, Phys. Rev. B 102, 241115(R) (2020).

[111] O. Hart, G. De Tomasi, and C. Castelnovo, From compact localized states to many-body scars in the random quantum comb, Phys. Rev. Res. 2, 043267 (2020).

[112] N. Shibata, N. Yoshioka, and H. Katsura, Onsager's Scars in Disordered Spin Chains, Phys. Rev. Lett. 124, 180604 (2020).

[113] C. Hubig, J. Haegeman, and U. Schollwöck, Error estimates for extrapolations with matrix-product states, Phys. Rev. B 97, 045125 (2018).

[114] E. Lieb, T. Schultz, and D. Mattis, Two soluble models of an antiferromagnetic chain, Ann. Phys. 16, 407 (1961).

[115] I. Affleck and E. H. Lieb, A proof of part of Haldane's conjecture on spin chains, Lett. Math. Phys. 12, 57 (1986).

[116] M. Oshikawa, Commensurability, Excitation Gap, and Topology in Quantum Many-Particle Systems on a Periodic Lattice, Phys. Rev. Lett. 84, 1535 (2000).

[117] M. B. Hastings, Lieb-Schultz-Mattis in higher dimensions, Phys. Rev. B 69, 104431 (2004).
[118] Y. Yao and M. Oshikawa, Generalized Boundary Condition Applied to Lieb-Schultz-Mattis-Type Ingappabilities and Many-Body Chern Numbers, Phys. Rev. X 10, 031008 (2020).

[119] Y. Fuji, Effective field theory for one-dimensional valencebond-solid phases and their symmetry protection, Phys. Rev. B 93, 104425 (2016).

[120] S. A. Parameswaran, A. M. Turner, D. P. Arovas, and A. Vishwanath, Topological order and absence of band insulators at integer filling in non-symmorphic crystals, Nat. Phys. 9, 299 (2013).

[121] H. Watanabe, H. C. Po, A. Vishwanath, and M. Zaletel, Filling constraints for spin-orbit coupled insulators in symmorphic and nonsymmorphic crystals, Proc. Natl. Acad. Sci. USA 112, 14551 (2015).

[122] Y. Ogata and H. Tasaki, Lieb-Schultz-Mattis type theorems for quantum spin chains without continuous symmetry, Commun. Math. Phys. 372, 951 (2019).

[123] Y. Ogata, Y. Tachikawa, and H. Tasaki, General LiebSchultz-Mattis type theorems for quantum spin chains, arXiv:2004.06458 (2020).

[124] Y. Yao and M. Oshikawa, Twisted Boundary Condition and Lieb-Schultz-Mattis Ingappability for Discrete Symmetries, Phys. Rev. Lett. 126, 217201 (2021).

[125] T. Hirano, H. Katsura, and Y. Hatsugai, Degeneracy and consistency condition for Berry phases: Gap closing under a local gauge twist, Phys. Rev. B 78, 054431 (2008).

[126] As already noted in Appendix B, for SPT phases protected by crystalline symmetry alone in $d<3$ dimensions, there are no anomalous edge states [4,95].

[127] M. Cheng, M. Zaletel, M. Barkeshli, A. Vishwanath, and P. Bonderson, Translational Symmetry and Microscopic Constraints on Symmetry-Enriched Topological Phases: A View from the Surface, Phys. Rev. X 6, 041068 (2016).

[128] D. Pérez-García, M. M. Wolf, M. Sanz, F. Verstraete, and J. I. Cirac, String Order and Symmetries in Quantum Spin Lattices, Phys. Rev. Lett. 100, 167202 (2008).

[129] D. Perez-Garcia, F. Verstraete, M. M. Wolf, and J. I. Cirac, Matrix product state representations, Quantum Inf. Comput. 7, 401 (2007).

[130] M. Fannes, B. Nachtergaele, and R. F. Werner, Finitely correlated states on quantum spin chains, Commun. Math. Phys. 144, 443 (1992).

[131] X. Chen, Z.-C. Gu, Z.-X. Liu, and X.-G. Wen, Symmetry protected topological orders and the group cohomology of their symmetry group, Phys. Rev. B 87, 155114 (2013).

[132] X. Chen, Z.-X. Liu, and X.-G. Wen, Two-dimensional symmetry-protected topological orders and their protected gapless edge excitations, Phys. Rev. B 84, 235141 (2011).

[133] S. Takayoshi, P. Pujol, and A. Tanaka, Field theory of symmetry-protected valence bond solid states in $(2+1)$ dimensions, Phys. Rev. B 94, 235159 (2016). 